XMWAS: R package for data integration and network analysis

\title{
XMWAS: an R package for data-driven integration and differential net- work analysis
}

\author{
Karan Uppal ${ }^{\star 1}$
}

Clinical Biomarkers Laboratory, Division of Pulmonary Medicine, Department of Medicine, Emory University, Atlanta, Georgia 30322, USA

E-mail:kuppal2@emory.edu

\section{Young-Mi Go}

Clinical Biomarkers Laboratory, Division of Pulmonary Medicine, Department of Medicine, Emory University, Atlanta, Georgia 30322, USA

$$
\text { Dean P. Jones }
$$

Clinical Biomarkers Laboratory, Division of Pulmonary Medicine, Department of Medicine, Emory University, Atlanta, Georgia 30322, USA

${ }^{1}$ To whom correspondence should be addressed 


\section{XMWAS: an $\mathbf{R}$ package for data-driven integration and differential net-} work analysis

Karan Uppal", Young-Mi Go and Dean P. Jones

Clinical Biomarkers Laboratory, Department of Medicine, Emory University, Atlanta, GA

*To whom correspondence should be addressed.

\section{Abstract}

Summary: Integrative omics is a central component of most systems biology studies. Computational methods are required for extracting meaningful relationships across different omics layers. Various tools have been developed to facilitate integration of paired heterogenous omics data; however most existing tools allow integration of only two omics datasets. Furthermore, existing data integration tools do not incorporate additional steps of identifying sub-networks or communities of highly connected entities and evaluating the topology of the integrative network under different conditions. Here we present xMWAS, an R package for data integration, network visualization, clustering, differential network analysis of data from biochemical and phenotypic assays, and two or more omics platforms.

Availability: https://sourceforge.net/projects/xmwas/

Contact: kuppal2@emory.edu

\section{Introduction}

Technological advances have led to a major paradigm shift where multi-assay molecular profiling of biological samples is increasingly being used to understand molecular mechanisms for diseases and host responses to environmental exposures (Hawkins 2010, Cancer Genome Atlas Network 2008). Most cellular processes in a biological system are dependent on complex molecular interactions (Barabasi 2011). Integrative omics allows researchers to address such complexity and answer challenging biological questions, such as function of genetic variants and unknown metabolites, mechanisms of gene regulation, signaling and metabolic pathway responses to infection and toxicity (Hawkins 2010, Chandler 2016, Uppal 2016).

Numerous data-driven/unsupervised and knowledge-based tools allow integration of data from different omics technologies and other molecular assays (Wanichthanarak 2015, Meng 2016). Most existing data integration tools allow integration of only two datasets and do not allow identification of community structure and evaluation of network changes between different conditions. Community detection reveals topological modules comprised of functionally related biomolecules (Barabasi 2011, Yang 2016). Differential network analysis (DiNA) allows characterization of 
xMWAS: $R$ package for data integration and network analysis

nodes that undergo changes in topological characteristics between different conditions, e.g. healthy vs disease (Lichtblau 2016).

To advance these capabilities, we present, xMWAS, an R package that provides an automated workflow for integrative analysis of more than two datasets, differential network analysis, and community detection to improve our understanding of complex molecular interactions and disease mechanisms.

A.

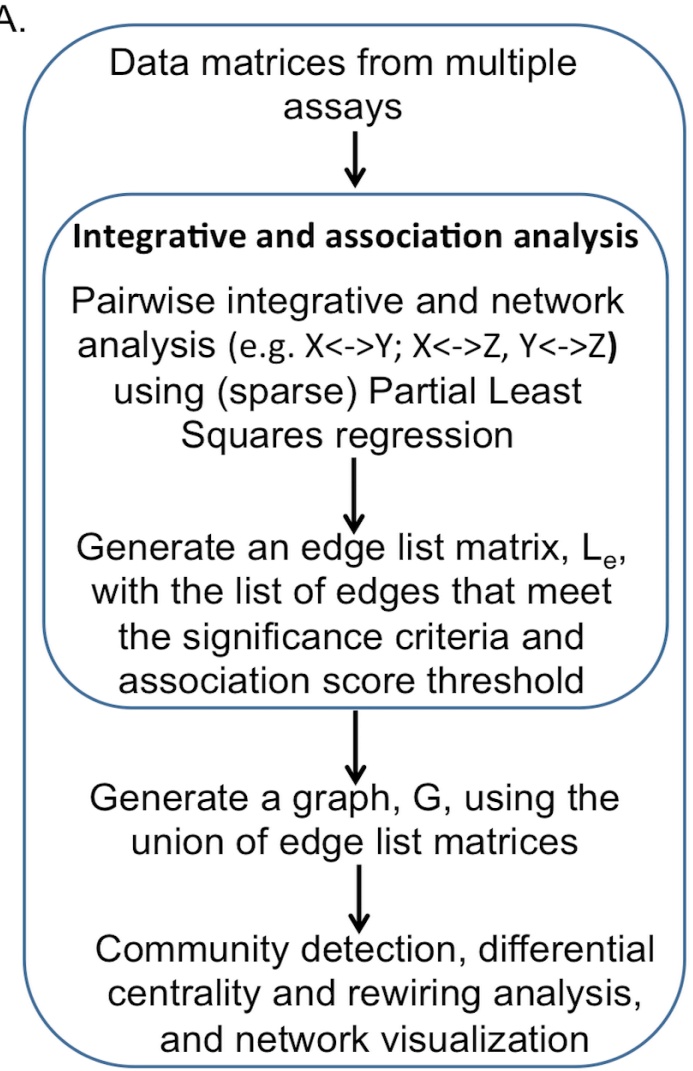

B.

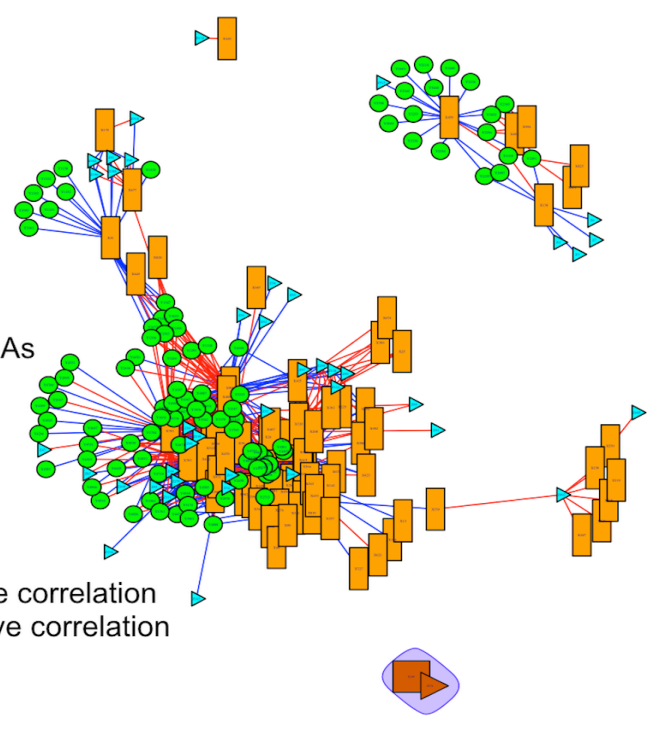

C.



Fig. 1. xMWAS workflow. A. Pairwise data integration and association analysis is performed using (sparse) partial least squares regression. The edge list matrices of significant associations from each pairwise comparison are merged to generate a global association network. Community detection is performed using the multilevel algorithm. B. Illustration of association network for the NCI60 dataset (Meng 2016): microRNAs (triangle), protein (circle) and transcripts (rectangle). C. Identification of communities in the NCI60 integrated network using the multilevel community detection algorithm.

\section{Implementation}

xMWAS provides an automated framework for integrative and differential network analysis. Figure 1A provides an overview of different stages of xMWAS. In stage one, xMWAS uses dimension reduction techniques such as Partial Least Squares (PLS), sparse Partial Least Squares (sPLS), and multilevel sparse Partial Least Squares (msPLS; for repeated measures) regression for 
pairwise integrative and association analysis between data matrices (Le Cao 2009, Liquet 2012, Gonzalez 2012). sPLS and msPLS methods perform simultaneous data integration and variable selection using a LASSO penalty for the loading vectors, which reduces the complexity of the networks (Liquet 2012). R package plsgenomics is used to determine the optimal number of latent components. The network() function in the mixOmics package is used to generate the association matrix, $A_{X Y}$, between matrices X and Y (Le Cao 2009, Gonzalez 2012). Student's t-test is used to evaluate the statistical significance of association scores. Only the associations that satisfy the user-defined thresholds, e.g. |association score $>0.7$ and $p$-value $<0.01$, are used for downstream analysis. The resulting graph, $\mathrm{G}_{\mathrm{i}}=(\mathrm{V}, \mathrm{E})$, where $\mathrm{V}$ is a set of nodes and $\mathrm{E}$ is a set of edges, is used to generate an edge list matrix, $\mathrm{L}_{\mathrm{i}}$, such that each row in $\mathrm{L}_{\mathrm{i}}$ corresponds to an edge between nodes $\mathrm{X}_{\mathrm{p}}$ and $\mathrm{Y}_{\mathrm{q}}$. The same process is repeated for generating edge list matrices from all pairwise association analyses between datasets, e.g. $\mathrm{L}_{\mathrm{i}}=\operatorname{cor}(\mathrm{X}, \mathrm{Y}) ; \mathrm{L}_{\mathrm{j}}=\operatorname{cor}(\mathrm{Y}, \mathrm{Z})$, and $\mathrm{L}_{\mathrm{k}}=\operatorname{cor}(\mathrm{Y}, \mathrm{Z})$.

In stage two, the union of the individual edge list matrices from pairwise integrative analysis of the $\mathrm{n}$ datasets is used to generate a combined edge list matrix, $\mathrm{L}_{\mathrm{e}}=\mathrm{U}_{i=1}^{n} L i$. Matrix $\mathrm{L}_{\mathrm{e}}$ is used to generate the integrative network graph, $G=(V, E)$, where $V$ corresponds to nodes and $E$ corresponds to edges or connections between the nodes, representing positive or negative associations between multiple datasets (Figure 1B). Network graphics are generated using the igraph package in $\mathrm{R}$.

In stage three, the multilevel community detection algorithm (Blondel 2008) is used to identify communities of nodes that are tightly connected with each other, but sparsely connected with the rest of the network (Figure 1C). Comparative studies for community detection algorithms show that the multilevel algorithm is suitable for both small and large networks with varying connectivity patterns (Yang 2016). The quality of the community structure is evaluated using the network modularity measure (Newman 2006).

Differential network analysis is performed using the differential betweenness centrality and differential eigenvector centrality methods to identify nodes that undergo changes in their topological characteristics (Odibat 2012, Lichtblau 2016). Additional description about the software input and output is provided in Supplementary Section S1.

\section{Example}

We tested xMWAS in a three-way integrative analysis using cytokine, transcriptome, and metabolome datasets from a recently published study to examine H1N1 influenza virus infection-altered metabolic response in mouse lung (Chandler 2016). For comparisons, we used data from all samples (Supplementary Figure S1A), only control samples (Supplementary Figure S1B), and only H1N1 influenza samples (Supplementary Figure S1C). Supplementary Section S2 shows that the various stages of xMWAS capture biologically meaningful information and provide deeper insights into the underlying biology, which cannot be obtained by analyzing and exploring the different layers individually.

\section{Conclusion}

xMWAS provides a platform-independent framework for integrative network analysis of two or more datasets, identification of modules of functionally related biomolecules, and differential network analysis. The results show that xMWAS can improve our understanding of disease pathophysiology and complex molecular interactions across various functional levels. 


\section{Acknowledgements}

The authors acknowledge members of the Clinical Biomarkers Laboratory, Emory University for testing and suggesting improvements to the software.

\section{Funding}

This project was funded by National Institutes of Health grants, ES025632, ES023485, ES019776, OD018006, HL095479, EY022618. The project was also funded in part by federal funds from the US National Institute of Allergy and Infectious Diseases, National Institutes of Health, Department of Health and Human Services under contract \# HHSN272201200031C.

Conflict of Interest: none declared.

\section{References}

Barabasi, A.L. et al. (2011) Network medicine: a network-based approach to human disease. Nat Rev Genet, 12, 56-68.

Blondel VD. et al. (2008) Fast unfolding of communities in large networks. Journal of Statistical Mechanics: Theory and Experiment, 2008, P10008.

Cancer Genome Atlas Research Network. (2008) Comprehensive genomic characterization defines human glioblastoma genes and core pathways. Nature, 455,1061-1068.

Chandler JD. et al. (2016) Metabolic pathways of lung inflammation revealed by high-resolution metabolomics (HRM) of H1N1 influenza virus infection in mice. Am J Physiol Regul Integr Comp Physiol,311, R906-R916.

Gonzalez, I. et al. (2012) Visualising associations between paired 'omics' data sets. BioData Min, $5,19$.

Hawkins, R.D. et al. (2010) Next-generation genomics: an integrative approach. Nat Rev Genet, 11,476-486.

Le Cao, K.A. et al. (2009) integrOmics: an R package to unravel relationships between two omics datasets. Bioinformatics, 25, 2855-2856.

Lichtblau, Y. et al. (2016) Comparative assessment of differential network analysis methods. Brief Bioinform 2016.

Liquet, B. et al. (2012) A novel approach for biomarker selection and the integration of repeated measures experiments from two assays. BMC Bioinformatics, 13, 325.

Meng C. et al.. (2016) Dimension reduction techniques for the integrative analysis of multi-omics data. Brief Bioinform, 17, 628-41.

Newman, M.E. (2006) Modularity and community structure in networks. Proc Natl Acad Sci U S $A, 103,8577-8582$.

Odibat, O. and Reddy, C.K. (2012) Ranking differential hubs in gene co-expression networks. $J$ Bioinform Comput Biol, 10, 1240002.

Uppal, K. et al. (2016) Computational Metabolomics: A Framework for the Million Metabolome. Chem Res Toxicol, 29, 1956-1975.

Yang, Z.. et al. (2016) A Comparative Analysis of Community Detection Algorithms on Artificial Networks. Sci Rep, 6,30750.

Wanichthanarak K. et al. (2015) Genomic, Proteomic, and Metabolomic Data Integration Strategies. Biomark Insights, 10, 1-6. 
XMWAS: $R$ package for data integration and network analysis

\title{
Supplementary Material
}

\section{xMWAS: an R package for data-driven integration and differential network analysis}

\author{
Karan Uppal*2
}

Clinical Biomarkers Laboratory, Division of Pulmonary Medicine, Department of Medicine, Emory University, Atlanta, Georgia 30322, USA

E-mail: kuppal2@emory.edu

\section{Young-Mi Go}

Clinical Biomarkers Laboratory, Division of Pulmonary Medicine, Department of Medicine, Emory University, Atlanta, Georgia 30322, USA

Dean P. Jones

Clinical Biomarkers Laboratory, Division of Pulmonary Medicine, Department of Medicine, Emory University, Atlanta, Georgia 30322, USA

\footnotetext{
${ }^{2}$ To whom correspondence should be addressed
} 
xMWAS: $R$ package for data integration and network analysis

\section{Supplementary Section S1. Description of input and output for xMWAS}

The main input parameters include file paths or data matrices of input datasets, file with phenotypic labels (e.g. case, control), integration method ("pls", "spls"), threshhold for association score, and statistical significance. The software is designed to work with data from different sources and does not utilize any knowledgebase information for integration. For metabolomics studies, the software is designed to work with both targeted and untargeted datasets and does not require metabolite identification prior to integration. The output includes PDF of integrative network with cluster assignments, text files for association matrices and cluster assignments for each node and their centrality scores, and a GML format file that can be used for visualization with tools such as Cytoscape (Shannon 2003).
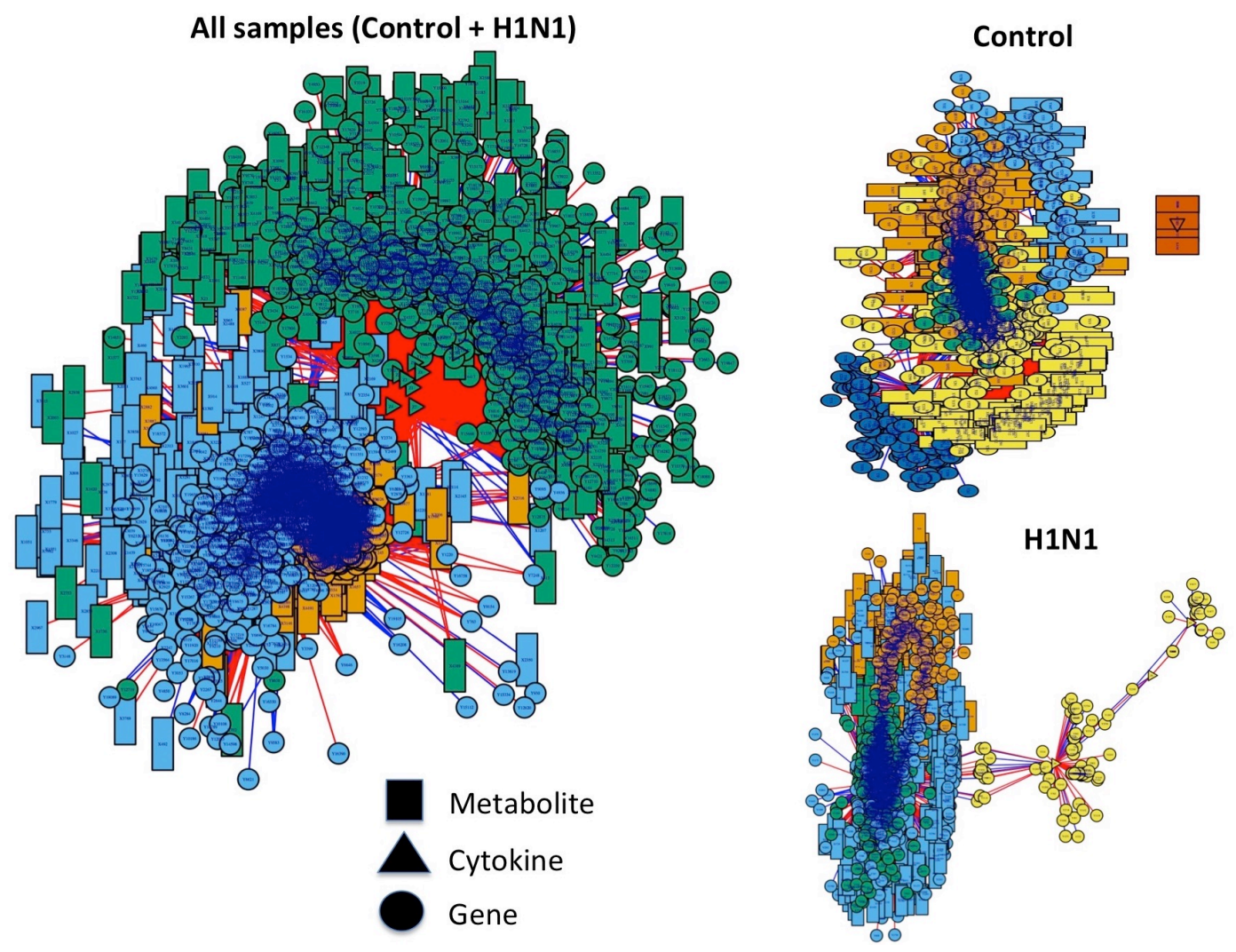

Figure S1. Integrative network analysis of cytokine, metabolome and transcriptome datasets from a study of H1N1 virus infection of mice. A. All samples; B. Only control samples; C. Only H1N1 infected samples. The results show that xMWAS analysis of the complex omics arrays allows identification and visualization of metabolite and transcript correlations with cytokines. The analysis further discriminates between correlations observed in controls from those observed in H1N1 virus infected mice and allows identification of nodes that undergo network changes. Details are provided in Supplementary Section S2.

\section{Supplementary Section S2. Evaluation results using data from the H1N1 virus infection of mice}


Pathway analysis of metabolic features $(\mathrm{m} / \mathrm{z}$ and retention time) and genes that were significantly associated with cytokines at $p<0.05$ across all samples was performed using Mummichog and MetaCore, respectively. Pathways related to immune response, lipid metabolism, amino acids metabolism, nitrogen metabolism, drug metabolism, vitamin metabolism, cytoskeleton remodeling, cell signaling, and energy metabolism were significantly enriched (Supplementary Tables $\mathrm{S} 1$ and S2). Previous studies have shown the role of these pathways in regulation of immune response and inflammation (Miyake 2000, Fortin 2009, Sadik 2012, Yin 2014, Chandler 2016)

Differential centrality analysis between control vs H1N1 samples showed dramatic change in the centrality of cytokines as well as genes and metabolites involved in immune response and defense mechanism (Supplementary Tables S3-6). Cytokines are known to be involved in the recruitment of the inflammatory cells and influence the adaptive immune response during influenza (Vareille 2011, Liu 2016, Chandler 2016).

Multilevel community detection identified four clusters in the integrative network generated using only H1N1 samples (Supplementary Figure S1C). Cluster 2 (blue), which comprised of four cytokines, 830 genes, and 437 metabolic features, was evaluated for significantly enriched pathways and biological processes. Pathway analysis of the metabolic features in this cluster showed enrichment of similar pathways as in the cytokine $\mathrm{x}$ metabolome association analysis across all samples described above (Supplementary Table S7). Pathway and process enrichment analysis using MetaCore showed enrichment of genes involved in immune responses, protein folding, signaling mechanisms, and various biological processes related to cilium movement, organization, assembly and organization (Supplementary Tables S8-S10). Previous studies have shown that the airway epithelium acts as a first layer of defense mechanism against respiratory viruses and that the respiratory viruses can lead to ciliary impairment (Vareille 2011). 
XMWAS: $R$ package for data integration and network analysis

Supplementary Table S1. Pathway analysis of metabolic features ( $m / z$ and retention time) that were found to be significantly associated with the six cytokines at $p<0.05$ using Mummichog

\begin{tabular}{|c|c|c|c|c|c|c|}
\hline Pathway Name & IL-1beta & IL-6 & IL-10 & TNFalpha & IP-1 & IFNgamma \\
\hline Carnitine shuttle & $\mathrm{x}$ & $\mathrm{x}$ & $\mathrm{x}$ & $\mathrm{x}$ & $\mathrm{x}$ & \\
\hline $\begin{array}{l}\text { Glycosphingolipid me- } \\
\text { tabolism }\end{array}$ & $\mathrm{x}$ & $\mathrm{x}$ & $\mathrm{x}$ & $\mathrm{x}$ & $\mathrm{x}$ & \\
\hline $\begin{array}{l}\text { Vitamin } D_{3} \text { (cholecal- } \\
\text { ciferol) metabolism }\end{array}$ & $\mathrm{x}$ & $\mathrm{x}$ & $\mathrm{x}$ & $\mathrm{x}$ & $\mathrm{x}$ & \\
\hline $\begin{array}{l}\text { Vitamin } B_{6} \text { (pyridox- } \\
\text { ine) metabolism }\end{array}$ & & $\mathrm{x}$ & & $\mathrm{x}$ & $\mathrm{x}$ & $\mathrm{x}$ \\
\hline Biopterin metabolism & $\mathrm{x}$ & $\mathrm{x}$ & & $\mathrm{x}$ & $\mathrm{x}$ & \\
\hline $\begin{array}{c}\text { Saturated fatty acids } \\
\text { beta-oxidation }\end{array}$ & & $\mathrm{x}$ & & $\mathrm{x}$ & $\mathrm{x}$ & \\
\hline Vitamin E metabolism & $\mathrm{x}$ & & $\mathrm{x}$ & $\mathrm{x}$ & & \\
\hline Nitrogen metabolism & & $\mathrm{x}$ & & & $\mathrm{x}$ & \\
\hline $\begin{array}{l}\text { Urea cycle/amino } \\
\text { group metabolism }\end{array}$ & & & & & $\mathrm{x}$ & $\mathrm{x}$ \\
\hline $\begin{array}{l}\text { Drug metabolism - } \\
\text { cytochrome P450 }\end{array}$ & & & & & $\mathrm{x}$ & $\mathrm{x}$ \\
\hline $\begin{array}{c}\text { Tryptophan metabo- } \\
\text { lism }\end{array}$ & & & & & $\mathrm{x}$ & $\mathrm{x}$ \\
\hline $\begin{array}{c}\text { Arginine and proline } \\
\text { metabolism }\end{array}$ & & & & & & $\mathrm{x}$ \\
\hline Bile acid biosynthesis & & & & $\mathrm{x}$ & & \\
\hline Fatty acid metabolism & & & $\mathrm{x}$ & & & \\
\hline $\begin{array}{c}\text { Limonene and pinene } \\
\text { degradation }\end{array}$ & & & $\mathrm{x}$ & & & \\
\hline Linoleate metabolism & & & $\mathrm{x}$ & & & \\
\hline Tyrosine metabolism & & $\mathrm{x}$ & & & & \\
\hline
\end{tabular}


Supplementary Table S2. Pathway analysis of genes that were found to be significantly associated with the six cytokines at $p<0.05$ using MetaCore

\begin{tabular}{|c|c|c|c|c|c|c|}
\hline Pathway Name & IL-1beta & IL-6 & IL-10 & TNFalpha & IP-1 & IFNgamma \\
\hline $\begin{array}{c}\text { Immune response_Alternative } \\
\text { complement pathway }\end{array}$ & $\mathrm{x}$ & $\mathrm{x}$ & $\mathrm{x}$ & $\mathrm{x}$ & $\mathrm{x}$ & $\mathrm{x}$ \\
\hline GTP metabolism & $\mathrm{x}$ & $\mathrm{x}$ & $\mathrm{x}$ & $\mathrm{x}$ & $\mathrm{x}$ & \\
\hline $\begin{array}{l}\text { Cytoskeleton remodel- } \\
\text { ing_TGF, WNT and cyto- } \\
\text { skeletal remodeling }\end{array}$ & $\mathrm{x}$ & $\mathrm{x}$ & & $\mathrm{x}$ & $\mathrm{x}$ & $\mathrm{x}$ \\
\hline $\begin{array}{l}\text { Cytoskeleton remodel- } \\
\text { ing_Cytoskeleton remodel- } \\
\text { ing }\end{array}$ & $\mathrm{x}$ & $\mathrm{x}$ & & $\mathrm{x}$ & $\mathrm{x}$ & $\mathrm{x}$ \\
\hline $\begin{array}{l}\text { Alternative complement cas- } \\
\text { cade disruption in age- } \\
\text { related macular degeneration }\end{array}$ & $\mathrm{x}$ & $\mathrm{x}$ & & $\mathrm{x}$ & $\mathrm{x}$ & $\mathrm{x}$ \\
\hline ATP metabolism & $\mathrm{x}$ & $\mathrm{x}$ & & $\mathrm{x}$ & $\mathrm{x}$ & \\
\hline $\begin{array}{l}\text { Immune response_Lectin in- } \\
\text { duced complement pathway }\end{array}$ & & $\mathrm{x}$ & $\mathrm{x}$ & & $\mathrm{x}$ & $\mathrm{x}$ \\
\hline $\begin{array}{l}\text { Regulation of lipid metabo- } \\
\text { lism_Regulation of lipid me- } \\
\text { tabolism by niacin and iso- } \\
\text { prenaline }\end{array}$ & $\mathrm{x}$ & $\mathrm{x}$ & & $\mathrm{x}$ & & \\
\hline $\begin{array}{c}\text { Development_Oligodendrocyte } \\
\text { differentiation from adult } \\
\text { stem cells }\end{array}$ & & $\mathrm{x}$ & & & $\mathrm{x}$ & $\mathrm{x}$ \\
\hline $\begin{array}{c}\text { Cell adhesion_Chemokines } \\
\text { and adhesion }\end{array}$ & $\mathrm{x}$ & & & $\mathrm{x}$ & & \\
\hline $\begin{array}{l}\text { Expression targets of Tissue } \\
\text { factor signaling in cancer }\end{array}$ & $\mathrm{x}$ & & & $\mathrm{x}$ & & \\
\hline Cell adhesion_ECM remodel- & & $\mathrm{x}$ & & & & $\mathrm{x}$ \\
\hline $\begin{array}{c}\text { Immune response_Classical } \\
\text { complement pathway }\end{array}$ & & & $\mathrm{x}$ & & & $\mathrm{x}$ \\
\hline $\begin{array}{c}\text { Development_Regulation of } \\
\text { epithelial-to-mesenchymal } \\
\text { transition (EMT) }\end{array}$ & & & & & $\mathrm{x}$ & $\mathrm{x}$ \\
\hline $\begin{array}{l}\text { Protein folding and matura- } \\
\text { tion Insulin processing }\end{array}$ & & & $\mathrm{x}$ & & & \\
\hline $\begin{array}{l}\text { Glucocorticoid-induced eleva- } \\
\text { tion of intraocular pressure } \\
\text { as glaucoma risk factor }\end{array}$ & & & & & & $\mathrm{x}$ \\
\hline $\begin{array}{c}\text { Inhibition of neutrophil migra- } \\
\text { tion by proresolving lipid } \\
\text { mediators in COPD }\end{array}$ & & & & & & $\mathrm{x}$ \\
\hline
\end{tabular}


xMWAS: $R$ package for data integration and network analysis

Supplementary Table S3. Eigenvector centrality measures for cytokines with delta centrality (control vs H1N1) greater than 0.3

\begin{tabular}{lllll}
\hline Name & All samples & Control & H1N1 & $\begin{array}{l}\text { Delta } \\
\text { (Control vs H1N1) }\end{array}$ \\
\hline IL-10 & 0.61 & 0.00 & 1.00 & 1.00 \\
IL-1beta & 0.66 & 0.00 & 0.98 & 0.98 \\
TNF-alpha & 0.66 & 0.00 & 0.88 & 0.88 \\
IL-6 & 0.34 & 0.50 & 0.00 & 0.50 \\
\hline
\end{tabular}


Supplementary Table S4. Pathway analysis of metabolic features with delta centrality (control vs H1N1) greater than 0.3 using Mummichog

\begin{tabular}{|c|c|c|c|}
\hline Pathways & Overlap_size & Pathway_size & $p$ \\
\hline Carnitine shuttle & 16 & 18 & 0.00065 \\
\hline $\begin{array}{c}\text { Vitamin D3 (cholecalciferol) } \\
\text { metabolism }\end{array}$ & 10 & 10 & 0.00065 \\
\hline $\begin{array}{c}\text { Phytanic acid peroxisomal } \\
\text { oxidation }\end{array}$ & 4 & 4 & 0.00115 \\
\hline $\begin{array}{l}\text { De novo fatty acid biosynthe- } \\
\text { sis }\end{array}$ & 8 & 14 & 0.00134 \\
\hline Leukotriene metabolism & 11 & 23 & 0.00174 \\
\hline $\begin{array}{c}\text { Valine, leucine and isoleucine } \\
\text { degradation }\end{array}$ & 10 & 22 & 0.00278 \\
\hline Fatty acid activation & 6 & 11 & 0.00288 \\
\hline Fatty Acid Metabolism & 6 & 11 & 0.00288 \\
\hline Vitamin E metabolism & 7 & 15 & 0.00474 \\
\hline $\begin{array}{c}\text { Squalene and cholesterol bio- } \\
\text { synthesis }\end{array}$ & 7 & 15 & 0.00474 \\
\hline Pyruvate Metabolism & 7 & 15 & 0.00474 \\
\hline $\begin{array}{c}\text { Omega-6 fatty acid metabo- } \\
\text { lism }\end{array}$ & 2 & 2 & 0.01496 \\
\hline $\begin{array}{c}\text { Benzoate degradation via } \\
\text { CoA ligation }\end{array}$ & 2 & 2 & 0.01496 \\
\hline $\begin{array}{c}\text { Fatty acid oxidation, peroxi- } \\
\text { some }\end{array}$ & 2 & 2 & 0.01496 \\
\hline $\begin{array}{l}\text { Di-unsaturated fatty acid beta- } \\
\text { oxidation }\end{array}$ & 2 & 2 & 0.01496 \\
\hline $\begin{array}{c}\text { Prostaglandin formation from } \\
\text { arachidonate }\end{array}$ & 14 & 40 & 0.01604 \\
\hline Biopterin metabolism & 5 & 12 & 0.01882 \\
\hline Bile acid biosynthesis & 16 & 47 & 0.01928 \\
\hline $\begin{array}{c}\text { Glycosphingolipid metabo- } \\
\text { lism }\end{array}$ & 8 & 22 & 0.02174 \\
\hline $\begin{array}{l}\text { Vitamin B5 - CoA biosynthe- } \\
\text { sis from pantothenate }\end{array}$ & 3 & 6 & 0.02583 \\
\hline Tryptophan metabolism & 16 & 49 & 0.03124 \\
\hline $\begin{array}{c}\text { Saturated fatty acids beta- } \\
\text { oxidation }\end{array}$ & 2 & 3 & 0.03833 \\
\hline $\begin{array}{l}\text { Glyoxylate and Dicarboxylate } \\
\text { Metabolism }\end{array}$ & 3 & 7 & 0.04628 \\
\hline
\end{tabular}


Supplementary Table S5. Pathway analysis of genes with delta centrality (control vs H1N1) greater than 0.3 using MetaCore. Only the top 20 significant pathways at $F D R<0.1$ are shown here.

\begin{tabular}{|c|c|c|c|c|c|}
\hline Pathway Maps & Total & $p$ & FDR & $\begin{array}{c}\text { In } \\
\text { D } \\
\text { at } \\
\mathbf{a} \\
\end{array}$ & $\begin{array}{c}\text { Network Objects } \\
\text { from } \\
\text { Active Data }\end{array}$ \\
\hline $\begin{array}{l}\text { Expression targets of Tissue } \\
\text { factor signaling in cancer }\end{array}$ & 22 & $\begin{array}{c}2.429 \mathrm{E}- \\
04\end{array}$ & $\begin{array}{c}5.928 \mathrm{E}- \\
02\end{array}$ & 5 & $\begin{array}{l}\text { Tissue factor, } \\
\text { PAR2, NFKBIE, } \\
\text { PLAU (UPA), } \\
\text { CSF1 }\end{array}$ \\
\hline $\begin{array}{c}\text { Signal transduction_Soluble } \\
\text { CXCL16 signaling }\end{array}$ & 49 & $\begin{array}{c}3.036 \mathrm{E}- \\
04\end{array}$ & $\begin{array}{c}5.928 \mathrm{E}- \\
02\end{array}$ & 7 & $\begin{array}{c}\text { STAT3, HIF1A, I- } \\
\text { kB, JAK2, P- } \\
\text { selectin, CD163, } \\
\text { NF-kB }\end{array}$ \\
\hline $\begin{array}{c}\text { Immune response_IL-12 sig- } \\
\text { naling pathway }\end{array}$ & 23 & $\begin{array}{c}3.036 \mathrm{E}- \\
04\end{array}$ & $\begin{array}{c}5.928 \mathrm{E}- \\
02\end{array}$ & 5 & $\begin{array}{l}\text { STAT3, IRF1, } \\
\text { JAK2, P-selectin, } \\
\text { Perforin }\end{array}$ \\
\hline $\begin{array}{l}\text { Immune response_IFN al- } \\
\text { pha/beta signaling pathway }\end{array}$ & 24 & $\begin{array}{c}3.752 \mathrm{E}- \\
04\end{array}$ & $\begin{array}{c}5.928 \mathrm{E}- \\
02\end{array}$ & 5 & $\begin{array}{r}\text { IRF1, STAT2, PTP- } \\
\text { 1B, PML, USP18 }\end{array}$ \\
\hline $\begin{array}{c}\text { Development_Leptin signal- } \\
\text { ing via JAK/STAT and } \\
\text { MAPK cascades }\end{array}$ & 25 & $\begin{array}{c}4.588 \mathrm{E}- \\
04\end{array}$ & $\begin{array}{c}5.928 \mathrm{E}- \\
02\end{array}$ & 5 & $\begin{array}{l}\text { STAT3, HIF1A, } \\
\text { JAK2, cPLA2, } \\
\text { PTP-1B }\end{array}$ \\
\hline $\begin{array}{c}\text { Platelet activation during } \\
\text { ADAM-TS13-deficient } \\
\text { thrombotic microangiopa- } \\
\text { thy development }\end{array}$ & 28 & $\begin{array}{c}7.946 \mathrm{E}- \\
04\end{array}$ & $\begin{array}{c}7.381 \mathrm{E}- \\
02\end{array}$ & 5 & $\begin{array}{c}\text { Calpain } 1(\mathrm{mu}), \mathrm{P}- \\
\text { selectin, Histone } \\
\text { H4, CD36, Fc } \\
\text { gamma RII alpha }\end{array}$ \\
\hline $\begin{array}{c}\text { Retinal ganglion cell damage } \\
\text { in glaucoma }\end{array}$ & 44 & $\begin{array}{c}1.059 \mathrm{E}- \\
03\end{array}$ & $\begin{array}{c}7.381 \mathrm{E}- \\
02\end{array}$ & 6 & $\begin{array}{l}\text { Calpain 1(mu), } \\
\text { TNF-R1, P2X7, } \\
\text { Sortilin, NF-kB, } \\
\text { MAP1LC3B }\end{array}$ \\
\hline $\begin{array}{c}\text { Myeloid-derived suppressor } \\
\text { cells and M2 macrophages } \\
\text { in cancer }\end{array}$ & 64 & $\begin{array}{c}1.560 \mathrm{E}- \\
03\end{array}$ & $\begin{array}{c}7.381 \mathrm{E}- \\
02\end{array}$ & 7 & $\begin{array}{l}\text { STAT3, IRF8, } \\
\text { ARG1, HIF1A, } \\
\text { NF-kB, IL4RA, } \\
\text { CSF1 }\end{array}$ \\
\hline Chemotaxis_CCR1 signaling & 48 & $\begin{array}{c}1.684 \mathrm{E}- \\
03\end{array}$ & $\begin{array}{c}7.381 \mathrm{E}- \\
02\end{array}$ & 6 & $\begin{array}{l}\text { STAT3, I-kB, } \\
\text { cPLA2, CCR1, } \\
\text { NF-kB, CCL7 }\end{array}$ \\
\hline $\begin{array}{c}\text { Bacterial infections in CF } \\
\text { airways }\end{array}$ & 48 & $\begin{array}{c}1.684 \mathrm{E}- \\
03\end{array}$ & $\begin{array}{c}7.381 \mathrm{E}- \\
02\end{array}$ & 6 & $\begin{array}{c}\text { IRF1, IKK-epsilon, } \\
\text { MyD88, JAK2, } \\
\text { Cathepsin S, NF- } \\
\text { kB }\end{array}$ \\
\hline $\begin{array}{c}\text { Immune response_CD40 sig- } \\
\text { naling }\end{array}$ & 65 & $\begin{array}{c}1.709 \mathrm{E}- \\
03\end{array}$ & $\begin{array}{c}7.381 \mathrm{E}- \\
02\end{array}$ & 7 & $\begin{array}{l}\text { STAT3, IRF1, } \\
\text { CCL2, I-kB, } \\
\text { JAK2, Lyn, NF- }\end{array}$ \\
\hline
\end{tabular}


xMWAS: $R$ package for data integration and network analysis

\begin{tabular}{|c|c|c|c|c|c|}
\hline & & & & & $\mathrm{kB}$ \\
\hline $\begin{array}{c}\text { SLE genetic marker-specific } \\
\text { pathways in antigen- } \\
\text { presenting cells (APC) }\end{array}$ & 84 & $\begin{array}{c}1.813 \mathrm{E}- \\
03\end{array}$ & $\begin{array}{c}7.381 \mathrm{E}- \\
02\end{array}$ & 8 & $\begin{array}{c}\text { IRF1, IKK-epsilon, } \\
\text { IRF8, MyD88, I- } \\
\text { kB, MICB, NF- } \\
\text { kB, Fc gamma } \\
\text { RII alpha }\end{array}$ \\
\hline $\begin{array}{l}\text { G-protein signaling_G- } \\
\text { Protein alpha-q signaling } \\
\text { cascades }\end{array}$ & 34 & $\begin{array}{c}1.973 \mathrm{E}- \\
03\end{array}$ & $\begin{array}{c}7.381 \mathrm{E}- \\
02\end{array}$ & 5 & $\begin{array}{c}\text { I-kB, PKC-epsilon, } \\
\text { RGS3, NF-kB, } \\
\text { LARG }\end{array}$ \\
\hline $\begin{array}{c}\text { Development_GM-CSF sig- } \\
\text { naling }\end{array}$ & 50 & $\begin{array}{c}2.085 \mathrm{E}- \\
03\end{array}$ & $\begin{array}{c}7.381 \mathrm{E}- \\
02\end{array}$ & 6 & $\begin{array}{l}\text { STAT3, CCL2, I- } \\
\text { kB, JAK2, Lyn, } \\
\text { NF-kB }\end{array}$ \\
\hline $\begin{array}{l}\text { Transcription_Role of Akt in } \\
\text { hypoxia induced HIF1 ac- } \\
\text { tivation }\end{array}$ & 21 & $\begin{array}{c}2.118 \mathrm{E}- \\
03\end{array}$ & $\begin{array}{c}7.381 \mathrm{E}- \\
02\end{array}$ & 4 & $\begin{array}{c}\text { Heme oxygenase 1, } \\
\text { HIF1A, GLUT1, } \\
\text { PGK1 }\end{array}$ \\
\hline $\begin{array}{l}\text { Immune re- } \\
\text { sponse_Inflammasome in } \\
\text { inflammatory response }\end{array}$ & 35 & $\begin{array}{c}2.253 \mathrm{E}- \\
03\end{array}$ & $\begin{array}{c}7.381 \mathrm{E}- \\
02\end{array}$ & 5 & $\begin{array}{l}\text { TNF-R1, P2X7, I- } \\
\text { kB, Nod1, NF-kB }\end{array}$ \\
\hline $\begin{array}{c}\text { Development_Angiopoietin - } \\
\text { Tie2 signaling }\end{array}$ & 35 & $\begin{array}{c}2.253 \mathrm{E}- \\
03\end{array}$ & $\begin{array}{c}7.381 \mathrm{E}- \\
02\end{array}$ & 5 & $\begin{array}{c}\text { STAT3, I-kB, } \\
\text { GRB7, NF-kB, } \\
\text { NCK1 }\end{array}$ \\
\hline $\begin{array}{c}\text { Signal transduction_NF-kB } \\
\text { activation pathways }\end{array}$ & 51 & $\begin{array}{c}2.311 \mathrm{E}- \\
03\end{array}$ & $\begin{array}{c}7.381 \mathrm{E}- \\
02\end{array}$ & 6 & $\begin{array}{l}\text { MyD88, TNF-R1, } \\
\text { NF-kB2 (p52), I- } \\
\text { kB, NF-kB2 } \\
\text { (p100), NF-kB }\end{array}$ \\
\hline $\begin{array}{c}\text { Immune response_IL-15 sig- } \\
\text { naling via JAK-STAT cas- } \\
\text { cade }\end{array}$ & 22 & $\begin{array}{c}2.535 \mathrm{E}- \\
03\end{array}$ & $\begin{array}{c}7.381 \mathrm{E}- \\
02\end{array}$ & 4 & $\begin{array}{l}\text { STAT3, IL-15, } \\
\text { JAK2, STAT2 }\end{array}$ \\
\hline $\begin{array}{l}\text { LRRK2 and immune func- } \\
\text { tion in Parkinson's disease }\end{array}$ & 22 & $\begin{array}{c}2.535 \mathrm{E}- \\
03 \\
\end{array}$ & $\begin{array}{c}7.381 \mathrm{E}- \\
02 \\
\end{array}$ & 4 & $\begin{array}{l}\text { I-kB, JAK2, } \\
\text { FCGR3A, NF-kB }\end{array}$ \\
\hline
\end{tabular}


xMWAS: $R$ package for data integration and network analysis

Supplementary Table S6. Process networks enrichment analysis of genes with delta centrality (control vs H1N1) greater than 0.3 using MetaCore.

\begin{tabular}{|c|c|c|c|c|c|}
\hline Process networks & Total & $p$ & FDR & $\begin{array}{c}\text { In } \\
\text { Da- } \\
\text { ta }\end{array}$ & $\begin{array}{c}\text { Network Objects from } \\
\text { Active Data }\end{array}$ \\
\hline $\begin{array}{c}\text { Development_Regulation } \\
\text { of angiogenesis }\end{array}$ & 222 & $\begin{array}{c}5.828 \mathrm{E}- \\
05\end{array}$ & $\begin{array}{c}4.303 \mathrm{E}- \\
03\end{array}$ & 23 & $\begin{array}{c}\text { Ephrin-A, STAT3, IL-15, } \\
\text { CCL2, S2P, HIF1A, I- } \\
\text { kB, JAK2, HNF4- } \\
\text { alpha, Osteonectin, } \\
\text { Prolyl endopeptidase, } \\
\text { Ephrin-B2, Ephrin-B, } \\
\text { CCR1, Galpha(q)- } \\
\text { specific peptide } \\
\text { GPCRs, NF-kB, } \\
\text { Ephrin-A1, BTG1, Ihh, } \\
\text { PKC, NCK1, Hedge- } \\
\text { hog, Syndecan-3 }\end{array}$ \\
\hline $\begin{array}{l}\text { Immune re- } \\
\text { sponse_Antigen } \\
\text { presentation }\end{array}$ & 197 & $\begin{array}{c}8.134 \mathrm{E}- \\
05\end{array}$ & $\begin{array}{c}4.303 \mathrm{E}- \\
03\end{array}$ & 21 & $\begin{array}{c}\text { STAT3, PSMB1, RFX5, } \\
\text { TNF-R1, NF-kB2 } \\
\text { (p52), I-kB, JAK2, } \\
\text { PSMD3, IP-30, } \\
\text { STAT2, Cathepsin S, } \\
\text { MICB, AP-3 beta sub- } \\
\text { units, PSMD14, NF- } \\
\text { kB2 (p100), NFKBIE, } \\
\text { NFKBIA, PSMB2, } \\
\text { Legumain, NF-kB, } \\
\text { TAP2 (PSF2) }\end{array}$ \\
\hline $\begin{array}{l}\text { Immune re- } \\
\text { sponse_Phagosome in } \\
\text { antigen presentation }\end{array}$ & 243 & $\begin{array}{c}8.663 \mathrm{E}- \\
05\end{array}$ & $\begin{array}{c}4.303 \mathrm{E}- \\
03\end{array}$ & 24 & $\begin{array}{l}\text { PSMB1, Vinculin, NF- } \\
\text { kB2 (p52), I-kB, } \\
\text { PSMD3, CD63, Ca- } \\
\text { thepsin S, FCGR3A, } \\
\text { Lyn, Alpha-actinin, } \\
\text { PSMD14, NF-kB2 } \\
\text { (p100), NFKBIE, } \\
\text { NFKBIA, VCP, } \\
\text { PSMB2, Legumain, } \\
\text { NF-kB, RalGDS, Al- } \\
\text { pha-actinin 3, TAP2 } \\
\text { (PSF2), NCK1, FGR, } \\
\text { Fc gamma RII alpha }\end{array}$ \\
\hline $\begin{array}{c}\text { Immune re- } \\
\text { sponse_Phagocytosis }\end{array}$ & 222 & $\begin{array}{c}4.338 \mathrm{E}- \\
04\end{array}$ & $\begin{array}{c}1.616 \mathrm{E}- \\
02\end{array}$ & 21 & $\begin{array}{c}\text { IL-15, Myosin I, Vincu- } \\
\text { lin, NF-kB2 (p52), I- } \\
\text { kB, MyHC, CD63, } \\
\text { PKC-epsilon, }\end{array}$ \\
\hline
\end{tabular}




\begin{tabular}{|c|c|c|c|c|c|}
\hline & & & & & $\begin{array}{l}\text { FCGR3A, Lyn, Alpha- } \\
\text { actinin, NF-kB2 } \\
\text { (p100), NFKBIE, } \\
\text { NFKBIA, MRLC, NF- } \\
\text { kB, Alpha-actinin 3, } \\
\text { HDL proteins, NCK1, } \\
\text { FGR, Fc gamma RII } \\
\text { alpha }\end{array}$ \\
\hline $\begin{array}{c}\text { Inflammation_Interferon } \\
\text { signaling }\end{array}$ & 110 & $\begin{array}{c}7.078 \mathrm{E}- \\
04\end{array}$ & $\begin{array}{c}2.109 \mathrm{E}- \\
02\end{array}$ & 13 & $\begin{array}{l}\text { STAT3, IRF1, IRF8, } \\
\text { CCL2, JAK2, STAT2, } \\
\text { MICB, Pyrin (MEFV), } \\
\text { PTP-1B, TAP2 (PSF2), } \\
\text { PML, GBP2, CCL7 }\end{array}$ \\
\hline $\begin{array}{l}\text { Development_Blood } \\
\text { vessel morphogenesis }\end{array}$ & 228 & $\begin{array}{c}1.520 \mathrm{E}- \\
03\end{array}$ & $\begin{array}{c}3.632 \mathrm{E}- \\
02\end{array}$ & 20 & $\begin{array}{l}\text { STAT3, FOXC2, Furin, } \\
\text { PLGF, PDE, HIF1A, } \\
\text { Neuregulin 1, Gal- } \\
\text { pha(q)-specific nucleo- } \\
\text { tide-like GPCRs, } \\
\text { VEGF-D, Galpha(q)- } \\
\text { specific amine GPCRs, } \\
\text { MMP-19, CCR1, } \\
\text { FOXC1/2, ErbB4, } \\
\text { PGK1, Galpha(q)- } \\
\text { specific peptide } \\
\text { GPCRs, GRB7, NF- } \\
\text { kB, NCK1, FGF1 }\end{array}$ \\
\hline $\begin{array}{c}\text { Inflammation_Protein } \mathrm{C} \\
\text { signaling }\end{array}$ & 108 & $\begin{array}{c}1.932 \mathrm{E}- \\
03\end{array}$ & $\begin{array}{c}3.632 \mathrm{E}- \\
02\end{array}$ & 12 & $\begin{array}{l}\text { Tissue factor, MyD88, } \\
\text { NF-kB2 (p52), I-kB, } \\
\text { PAR2, Alpha-actinin, } \\
\text { NF-kB2 (p100), } \\
\text { NFKBIE, NFKBIA, } \\
\text { PLAU (UPA), NF-kB, } \\
\text { Alpha-actinin } 3\end{array}$ \\
\hline $\begin{array}{c}\text { Cell adhesion_Synaptic } \\
\text { contact }\end{array}$ & 184 & $\begin{array}{c}1.950 \mathrm{E}- \\
03\end{array}$ & $\begin{array}{c}3.632 \mathrm{E}- \\
02\end{array}$ & 17 & $\begin{array}{c}\text { Ephrin-A, K(+) channel, } \\
\text { subfamily J, Syntaxin } \\
\text { 1A, GluR3, Synapto- } \\
\text { tagmin, GABA-A re- } \\
\text { ceptor beta-2 subunit, } \\
\text { Ephrin-B2, Ephrin-B, } \\
\text { Ephrin-B2 (CTF2), Al- } \\
\text { pha-actinin, SHANK, } \\
\text { GABA-A receptor al- } \\
\text { pha-5 subunit, } \\
\text { SHANK2, Neuroligin, } \\
\text { Alpha-actinin 3, } \\
\text { NCK1, Neuroligin 3 }\end{array}$ \\
\hline
\end{tabular}


XMWAS: $R$ package for data integration and network analysis

Supplementary Table S7. Pathway analysis of the 437 metabolic features in cluster 2 in the H1N1 integrative network using Mummichog

\begin{tabular}{cccc}
\hline Pathways & Overlap_size & Pathway_size & $\boldsymbol{p}$ \\
\hline $\begin{array}{c}\text { Carnitine shuttle } \\
\text { Vitamin D } \text { (chole- } \\
\text { calciferol) metab- } \\
\text { olism }\end{array}$ & 9 & 18 & 0.00036 \\
$\begin{array}{c}\text { Linoleate metabo- } \\
\text { lism }\end{array}$ & 6 & 10 & 0.00037 \\
$\begin{array}{c}\text { Vitamin E metabo- } \\
\text { lism }\end{array}$ & 5 & 21 & 0.00050 \\
$\begin{array}{c}\text { Glycosphingolipid } \\
\text { metabolism }\end{array}$ & 6 & 15 & 0.00081 \\
$\begin{array}{c}\text { Bile acid biosynthe- } \\
\text { sis }\end{array}$ & 10 & 22 & 0.00100 \\
$\begin{array}{c}\text { Saturated fatty acids } \\
\text { beta-oxidation } \\
\text { Pyruvate Metabo- } \\
\text { lism }\end{array}$ & 2 & 47 & 0.00108 \\
$\begin{array}{c}\text { C21-steroid hor- } \\
\text { mone biosynthe- } \\
\text { sis and metabo- } \\
\text { lism }\end{array}$ & 3 & 3 & 0.00305 \\
\hline
\end{tabular}


xMWAS: $R$ package for data integration and network analysis

Supplementary Table S8. Pathway analysis of the 830 genes in cluster 2 in the H1N1 integrative network using MetaCore

\begin{tabular}{|c|c|c|c|c|c|}
\hline Pathway & Total & $p$ & FDR & $\begin{array}{c}\text { In } \\
\text { Data }\end{array}$ & $\begin{array}{c}\text { Network Objects from } \\
\text { Active Data }\end{array}$ \\
\hline $\begin{array}{l}\text { Protein folding and matura- } \\
\text { tion_Insulin processing }\end{array}$ & 20 & $2.16 \mathrm{E}-10$ & $1.45 \mathrm{E}-07$ & 9 & $\begin{array}{l}\text { Des }(64,65) \text { proinsulin, } \\
\text { Split }(32,33) \text { proinsulin, } \\
\text { Insulin processed, Di- } \\
\text { arginyl insulin, Proin- } \\
\text { sulin C-peptide, Split } \\
(64,65) \text { proinsulin, Insu- } \\
\text { lin, Proinsulin, } \\
\text { Des }(31,32) \text { proinsulin }\end{array}$ \\
\hline $\begin{array}{c}\text { Immune response_IL-15 sig- } \\
\text { naling via JAK-STAT cas- } \\
\text { cade }\end{array}$ & 22 & $1.20 \mathrm{E}-04$ & $3.02 \mathrm{E}-02$ & 5 & $\begin{array}{c}\text { STAT3, IL-15, JAK2, sIL- } \\
\text { 15RA, IL-15RA }\end{array}$ \\
\hline $\begin{array}{c}\text { Immune response_Lectin } \\
\text { induced complement } \\
\text { pathway }\end{array}$ & 50 & $1.38 \mathrm{E}-04$ & $3.02 \mathrm{E}-02$ & 7 & $\begin{array}{c}\mathrm{C} 2, \mathrm{C} 2 \mathrm{~b}, \mathrm{C} 4 \mathrm{a}, \mathrm{C} 4, \mathrm{C} 2 \mathrm{a}, \mathrm{C} 3 \\
\text { convertase }(\mathrm{C} 2 \mathrm{aC} 4 \mathrm{~b}), \\
\mathrm{C} 4 \mathrm{~b}\end{array}$ \\
\hline $\begin{array}{c}\text { Immune response_Classical } \\
\text { complement pathway }\end{array}$ & 53 & $2.00 \mathrm{E}-04$ & $3.02 \mathrm{E}-02$ & 7 & $\begin{array}{c}\mathrm{C} 2, \mathrm{C} 2 \mathrm{~b}, \mathrm{C} 4 \mathrm{a}, \mathrm{C} 4, \mathrm{C} 2 \mathrm{a}, \mathrm{C} 3 \\
\text { convertase }(\mathrm{C} 2 \mathrm{aC} 4 \mathrm{~b}), \\
\mathrm{C} 4 \mathrm{~b}\end{array}$ \\
\hline $\begin{array}{l}\text { Role of IL-23/ T17 patho- } \\
\text { genic axis in psoriasis }\end{array}$ & 54 & $2.25 \mathrm{E}-04$ & $3.02 \mathrm{E}-02$ & 7 & $\begin{array}{c}\text { STAT3, IL-15, sIL-15RA, } \\
\text { IL-15RA, CD86, } \\
\text { CD40(TNFRSF5), HLA- } \\
\text { C }\end{array}$ \\
\hline $\begin{array}{l}\text { Main growth factor signaling } \\
\text { cascades in multiple mye- } \\
\text { loma cells }\end{array}$ & 41 & $3.26 \mathrm{E}-04$ & $3.64 \mathrm{E}-02$ & 6 & $\begin{array}{c}\text { STAT3, MEK1(MAP2K1), } \\
\text { HIF1A, I-kB, FGFR3, } \\
\text { PI3K reg class IA }\end{array}$ \\
\hline $\begin{array}{l}\text { Cell adhesion_Integrin in- } \\
\text { side-out signaling in neu- } \\
\text { trophils }\end{array}$ & 77 & $3.84 \mathrm{E}-04$ & $3.67 \mathrm{E}-02$ & 8 & $\begin{array}{l}\text { MEK1(MAP2K1), ARF6, } \\
\text { LTBR1, MEK1/2, Cyto- } \\
\text { hesin1, Lyn, Hck, GRO- } \\
1\end{array}$ \\
\hline $\begin{array}{c}\text { Development_c-Kit ligand } \\
\text { signaling pathway during } \\
\text { hemopoiesis }\end{array}$ & 61 & 4.84E-04 & $4.06 \mathrm{E}-02$ & 7 & $\begin{array}{c}\text { STAT3, MGF, MEK1/2, } \\
\text { DOK1, JAK2, Lyn, } \\
\text { SLUG }\end{array}$ \\
\hline $\begin{array}{c}\text { Immune response_Fc gamma } \\
\text { R-mediated phagocytosis } \\
\text { in macrophages }\end{array}$ & 46 & $6.16 \mathrm{E}-04$ & 4.59E-02 & 6 & $\begin{array}{c}\text { ARF6, Vinculin, Lyn, Hck, } \\
\text { Profilin, PI3K reg class } \\
\text { IA }\end{array}$ \\
\hline $\begin{array}{c}\text { Immune response_CD40 } \\
\text { signaling }\end{array}$ & 65 & $7.15 \mathrm{E}-04$ & 4.79E-02 & 7 & $\begin{array}{c}\text { STAT3, I-kB, JAK2, Lyn, } \\
\text { A20, CD86, } \\
\text { CD40(TNFRSF5) }\end{array}$ \\
\hline $\begin{array}{c}\text { Development_NOTCH- } \\
\text { induced EMT }\end{array}$ & 19 & 8.19E-04 & $4.85 \mathrm{E}-02$ & 4 & $\begin{array}{l}\text { HEY1, VE-cadherin, } \\
\text { NOTCH4, SLUG }\end{array}$ \\
\hline $\begin{array}{c}\text { Signal transduction_Soluble } \\
\text { CXCL16 signaling }\end{array}$ & 49 & 8.69E-04 & $4.85 \mathrm{E}-02$ & 6 & $\begin{array}{l}\text { STAT3, HIF1A, I-kB, } \\
\text { MEK1/2, JAK2, CD163 }\end{array}$ \\
\hline Development_GM-CSF sig- & 50 & $9.68 \mathrm{E}-04$ & 4.99E-02 & 6 & STAT3, MEK1(MAP2K1), \\
\hline
\end{tabular}


bioRxiv preprint doi: https://doi.org/10.1101/122432; this version posted March 30, 2017. The copyright holder for this preprint (which was not certified by peer review) is the author/funder. All rights reserved. No reuse allowed without permission.

xMWAS: $R$ package for data integration and network analysis

naling

I-kB, JAK2, Lyn, Hck 
Supplementary Table S9. Process networks enrichment analysis of the 830 genes in cluster 2 in the H1N1 integrative network using MetaCore. Only the top 50 significantly enriched process networks are shown here.

\begin{tabular}{|c|c|c|c|c|c|}
\hline Process networks & Total & $p$ & FDR & $\begin{array}{c}\text { In } \\
\text { Data }\end{array}$ & $\begin{array}{c}\text { Network Objects from } \\
\text { Active Data }\end{array}$ \\
\hline $\begin{array}{l}\text { Immune re- } \\
\text { sponse_Phagosome in } \\
\text { antigen presentation }\end{array}$ & 243 & $\begin{array}{c}7.255 \mathrm{E}- \\
05\end{array}$ & $\begin{array}{c}1.110 \mathrm{E}- \\
02\end{array}$ & 22 & $\begin{array}{l}\text { PSMA4, NFKBIB, } \\
\text { ARF6, Vinculin, I-kB, } \\
\text { PSMD3, Profilin II, } \\
\text { Lyn, PSMA1, Alpha- } \\
\text { actinin, PSMD14, Hck, } \\
\text { TLR4, PSMA2, Profil- } \\
\text { in, PSMD12, HLA-C, } \\
\text { HLA-B, Alpha-actinin } \\
\text { 3, PI3K reg class IA } \\
\text { (p55-gamma), NCK1, } \\
\text { PI3K reg class IA }\end{array}$ \\
\hline $\begin{array}{l}\text { Immune re- } \\
\text { sponse_Antigen } \\
\text { presentation }\end{array}$ & 197 & $\begin{array}{c}2.965 \mathrm{E}- \\
04\end{array}$ & $\begin{array}{c}2.269 \mathrm{E}- \\
02\end{array}$ & 18 & $\begin{array}{l}\text { PSMA4, STAT3, } \\
\text { NFKBIB, RFX5, } \\
\text { PSMD8, I-kB, JAK2, } \\
\text { PSMD3, PSMA1, } \\
\text { HLA-E, PSMD14, } \\
\text { PSMA2, CD86, } \\
\text { CD40(TNFRSF5), } \\
\text { PSMD12, HLA-C, } \\
\text { HLA-B, CD209 }\end{array}$ \\
\hline $\begin{array}{c}\text { Inflammation_Interferon } \\
\text { signaling }\end{array}$ & 110 & $\begin{array}{c}6.422 \mathrm{E}- \\
04\end{array}$ & $\begin{array}{c}3.275 \mathrm{E}- \\
02\end{array}$ & 12 & $\begin{array}{c}\text { STAT3, } \\
\text { MEK1(MAP2K1), } \\
\text { MEK1/2, JAK2, MxA, } \\
\text { Pyrin (MEFV), ISG20, } \\
\text { CD86, SSAT, } \\
\text { CD40(TNFRSF5), } \\
\text { IFNAR2, SERPINB9 }\end{array}$ \\
\hline $\begin{array}{l}\text { Signal transduc- } \\
\text { tion_Insulin signaling }\end{array}$ & 171 & $\begin{array}{c}1.428 \mathrm{E}- \\
03\end{array}$ & $\begin{array}{c}5.461 \mathrm{E}- \\
02\end{array}$ & 15 & $\begin{array}{l}\text { Des }(64,65) \text { proinsulin, } \\
\text { MEK1(MAP2K1), } \\
\text { Split }(32,33) \text { proin- } \\
\text { sulin, Insulin pro- } \\
\text { cessed, JAK2, Diargi- } \\
\text { nyl insulin, Proinsulin } \\
\text { C-peptide, PYGM, } \\
\text { PKA-reg (cAMP- } \\
\text { dependent), Split } \\
\text { (64,65) proinsulin, In- } \\
\text { sulin, Proinsulin, } \\
\text { Des(31,32) proinsulin, } \\
\text { FADS2, PI3K reg class } \\
\text { IA }\end{array}$ \\
\hline
\end{tabular}


XMWAS: $R$ package for data integration and network analysis

\begin{tabular}{|c|c|c|c|c|c|}
\hline $\begin{array}{c}\text { Cell adhesion_Synaptic } \\
\text { contact }\end{array}$ & 184 & $\begin{array}{c}2.936 \mathrm{E}- \\
03\end{array}$ & $\begin{array}{c}7.812 \mathrm{E}- \\
02\end{array}$ & 15 & $\begin{array}{c}\text { Syntaxin 4, Synaptotag- } \\
\text { min II, Synaptotagmin, } \\
\text { Profilin II, Ephrin-B2, } \\
\text { Ephrin-B, Ephrin-B2 } \\
\text { (CTF2), Alpha-actinin, } \\
\text { SHANK, SynGAP, } \\
\text { Profilin, SHANK2, A1- } \\
\text { pha-actinin 3, Syntenin } \\
\text { 1, NCK1 }\end{array}$ \\
\hline $\begin{array}{c}\text { Immune re- } \\
\text { sponse_Phagocytosis }\end{array}$ & 222 & $\begin{array}{c}3.063 \mathrm{E}- \\
03\end{array}$ & $\begin{array}{c}7.812 \mathrm{E}- \\
02\end{array}$ & 17 & $\begin{array}{l}\text { IL-15, NFKBIB, Vincu- } \\
\text { lin, I-kB, MyHC, Pro- } \\
\text { filin II, Lyn, Alpha- } \\
\text { actinin, IL-15RA, Hck, } \\
\text { TLR4, Profilin, Alpha- } \\
\text { actinin 3, HDL pro- } \\
\text { teins, PI3K reg class IA } \\
\text { (p55-gamma), NCK1, } \\
\text { PI3K reg class IA }\end{array}$ \\
\hline
\end{tabular}


Supplementary Table S10. GO processes analysis of the 830 genes in cluster 2 in the H1N1 integrative network using MetaCore. Only the top 50 significantly enriched GO processes are shown here.

\begin{tabular}{|c|c|c|c|c|c|}
\hline GO Processes & Total & $p$ & FDR & $\begin{array}{c}\text { In } \\
\text { Data }\end{array}$ & $\begin{array}{c}\text { Network Objects from } \\
\text { Active Data }\end{array}$ \\
\hline $\begin{array}{l}\text { cell projection as- } \\
\text { sembly }\end{array}$ & 367 & $1.09 \mathrm{E}-20$ & $7.54 \mathrm{E}-17$ & 51 & $\begin{array}{c}\text { FLJ13305, DNAH5, Dystro- } \\
\text { phin, CCDC39, SPEF2, } \\
\text { ARMC4, DNAH1, } \\
\text { SPAG17, Vinculin, IQCG, } \\
\text { Rab8B, RABL2A, P2X7, } \\
\text { DZIP1L, ATP6M, MIP-T3, } \\
\text { LRRC50, MyHC, BBS9, } \\
\text { WHDC1, Dynein, ax- } \\
\text { onemal, heavy chains, } \\
\text { RPGRIP1L, Oxygen- } \\
\text { regulated protein 1, CFA54, } \\
\text { MYH10, Dynein 2, cyto- } \\
\text { plasmic, heavy chain, } \\
\text { CFA74, SPAG16, Alpha- } \\
\text { actinin, RFX3, RAP2C, } \\
\text { ABBA, KIAA1377(HIP5), } \\
\text { CLR3, CYFIP1, HYDIN, } \\
\text { TSGA2, CCDC176, } \\
\text { DNAI1, DZIP, FGD3, } \\
\text { Dynein, axonemal, inter- } \\
\text { mediate chains, NME5, } \\
\text { SNAP-29, Syntenin 1, } \\
\text { LRGUK, NCK1, KAD7, } \\
\text { C2orf39, RSPH4A, SNX10 }\end{array}$ \\
\hline cilium assembly & 226 & $1.98 \mathrm{E}-19$ & $5.75 \mathrm{E}-16$ & 39 & $\begin{array}{c}\text { FLJ13305, DNAH5, Dystro- } \\
\text { phin, CCDC39, SPEF2, } \\
\text { ARMC4, DNAH1, } \\
\text { SPAG17, IQCG, Rab8B, } \\
\text { RABL2A, DZIP1L, } \\
\text { ATP6M, MIP-T3, } \\
\text { LRRC50, BBS9, Dynein, } \\
\text { axonemal, heavy chains, } \\
\text { RPGRIP1L, Oxygen- } \\
\text { regulated protein 1, CFA54, } \\
\text { Dynein 2, cytoplasmic, } \\
\text { heavy chain, CFA74, } \\
\text { SPAG16, RFX3, } \\
\text { KIAA1377(HIP5), CLR3, } \\
\text { HYDIN, TSGA2, } \\
\text { CCDC176, DNAI1, DZIP, } \\
\text { Dynein, axonemal, inter- }\end{array}$ \\
\hline
\end{tabular}


xMWAS: $R$ package for data integration and network analysis

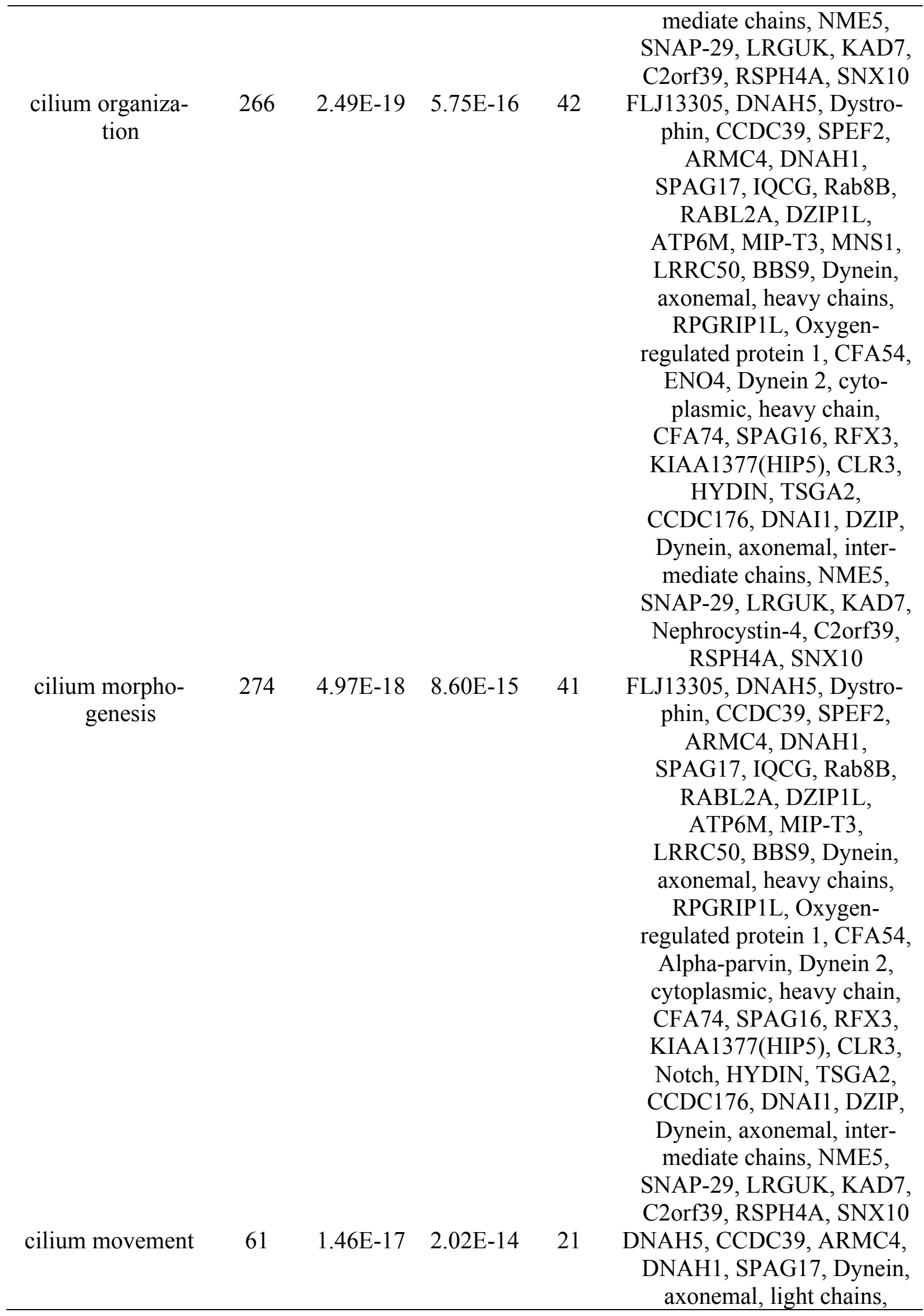




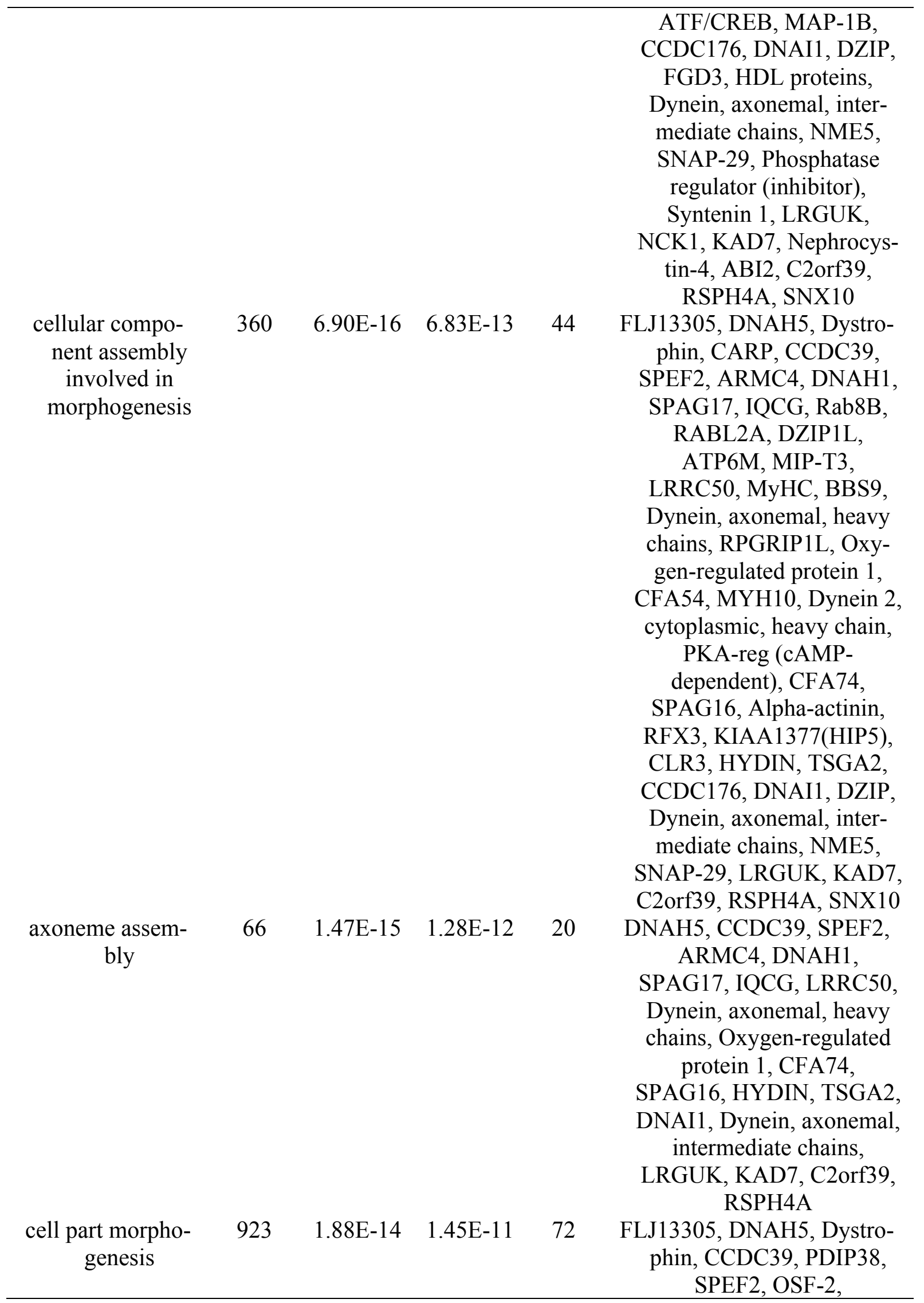


cell projection

morphogenesis
$889 \quad 2.84 \mathrm{E}-14 \quad 1.97 \mathrm{E}-11 \quad 70$

$x$ WAS: $R$ package for data integration and network analysis

\begin{tabular}{c}
\hline \\
PNPase(old-35), \\
MEK1(MAP2K1), \\
ARMC4, SLIT3, DNAH1, \\
SNX2, SNX1, Olfactory \\
receptor, SPAG17, Vincu- \\
lin, Dynein, axonemal, light \\
chains, IQCG, Rab8B, \\
RABL2A, MEK1/2, \\
B3GN1, ROBO1, Islet-1, \\
Galpha(q)-specific nucleo- \\
tide-like GPCRs, DZIP1L, \\
ATP6M, Rab-3, MIP-T3, \\
LRRC50, Ephrin-A recep- \\
tor 5, FGFR3, MyHC, \\
BBS9, Dynein, axonemal, \\
heavy chains, RPGRIP1L, \\
Oxygen-regulated protein \\
1, CFA54, Alpha-parvin, \\
MYH10, Ephrin-B2, \\
Ephrin-B, Dynein 2, cyto- \\
plasmic, heavy chain, \\
NPX1, CFA74, SPAG16, \\
RFX3, MENA, Ephrin-A \\
receptors, SHANK, \\
KIAA1377(HIP5), CLR3, \\
Notch, Galpha(q)-specific \\
peptide GPCRs, CYFIP1, \\
HYDIN, Rab-3A, TSGA2, \\
ATF/CREB, MAP-1B, \\
CCDC176, DNAI1, DZIP, \\
Dynein, axonemal, inter- \\
mediate chains, NME5, \\
SNAP-29, LRGUK, KAD7, \\
C2orf39, RSPH4A, SNX10 \\
FLJ13305, DNAH5, Dystro- \\
phin, CCDC39, SPEF2, \\
OSF-2, MEK1(MAP2K1), \\
ARMC4, SLIT3, DNAH1, \\
SNX2, SNX1, Olfactory \\
receptor, SPAG17, Vincu- \\
lin, Dynein, axonemal, light \\
chains, IQCG, Rab8B, \\
RABL2A, MEK1/2, \\
B3GN1, ROBO1, Islet-1, \\
Galpha(q)-specific nucleo- \\
tide-like GPCRs, DZIP1L, \\
ATP6M, Rab-3, MIP-T3, \\
\hline morphogenesis
\end{tabular}


cell morphogene- $1193 \quad 9.55 \mathrm{E}-14 \quad 6.02 \mathrm{E}-11 \quad 83$ sis
LRRC50, Ephrin-A receptor 5, FGFR3, MyHC, BBS9, Dynein, axonemal, heavy chains, RPGRIP1L, Oxygen-regulated protein 1, CFA54, Alpha-parvin, MYH10, Ephrin-B2, Ephrin-B, Dynein 2, cytoplasmic, heavy chain, NPX1, CFA74, SPAG16, RFX3, MENA, Ephrin-A receptors, SHANK, KIAA1377(HIP5), CLR3, Notch, Galpha(q)-specific peptide GPCRs, CYFIP1, HYDIN, Rab-3A, TSGA2, ATF/CREB, MAP-1B, CCDC176, DNAI1, DZIP, Dynein, axonemal, intermediate chains, NME5, SNAP-29, LRGUK, KAD7, C2orf39, RSPH4A, SNX10 FLJ13305, DNAH5, Dystrophin, CCDC39, SPEF2, OSF-2, MEK1(MAP2K1), ARMC4, SLIT3, DNAH1, SNX2, SNX1, Olfactory receptor, SPAG17, Vinculin, HEY1, Dynein, axonemal, light chains, IQCG, Rab8B, Ephrin-A receptor 1, HIF1A, RABL2A, FAT3, P2X7, NOTCH4, HES1, MEK1/2, B3GN1, ROBO1, Islet-1, Galpha(q)specific nucleotide-like GPCRs, DZIP1L, ATP6M, Rab-3, MIP-T3, LRRC50, Ephrin-A receptor 5, FGFR3, MyHC, PMCA2b, BBS9, Dynein, axonemal, heavy chains, RPGRIP1L, Oxygen-regulated protein 1, CFA54, Alpha-parvin, CAP2, MYH10, Ephrin-B2, Ephrin-B, Dynein 2, cytoplasmic, heavy chain, 
microtubule bundle formation

cilium or flagellum-dependent cell motility

cellular component morphogenesis
95

$2.91 \mathrm{E}-13 \quad 1.68 \mathrm{E}-10$

21

29

3.90E-13 1.77E-10

13

1316

3.93E-13 1.77E-10

87
NPX1, CFA74, SPAG16, Alpha-actinin, RFX3, SLUG, MENA, Ephrin-A receptors, SHANK, TMEM100, KIAA1377(HIP5), CLR3, Notch, Galpha(q)-specific peptide GPCRs, CYFIP1, HYDIN, Rab-3A, TSGA2, ATF/CREB, MAP-1B, CCDC176, DNAI1, DZIP, Dynein, axonemal, intermediate chains, NME5, SNAP-29, LRGUK, ILPIP, KAD7, C2orf39, RSPH4A, SNX10

DNAH5, CCDC39, SPEF2, ARMC4, DNAH1,

SPAG17, IQCG, LRRC50, Dynein, axonemal, heavy chains, Oxygen-regulated protein 1, CFA74,

SPAG16, HYDIN, TSGA2,

MAP-1B, DNAI1, Dynein, axonemal, intermediate chains, LRGUK, KAD7, C2orf39, RSPH4A

DNAH5, CCDC39, DNAH1, DNAH6, Dynein, axonemal, heavy chains, CFA54, SPAG16, DNAH11, RFX3, Sp17,

DNAH3, DNAH2, C2orf39

FLJ13305, DNAH5, Dystrophin, CARP, CCDC39, PDIP38, SPEF2, OSF-2, PNPase(old-35), MEK1(MAP2K1), ARMC4, SLIT3, DNAH1, SNX2, SNX1, Olfactory receptor, SPAG17, Vincu-

lin, HEY1, Dynein, axonemal, light chains, IQCG, Rab8B, Ephrin-A receptor 1, HIF1A, RABL2A, FAT3, P2X7, NOTCH4, HES1, MEK1/2, B3GN1, 
microtubule-based movement
ROBO1, Islet-1, Galpha(q)specific nucleotide-like GPCRs, DZIP1L, ATP6M, Rab-3, MIP-T3, LRRC50, Ephrin-A receptor 5, FGFR3, MyHC, PMCA2b, BBS9, Dynein, axonemal, heavy chains, RPGRIP1L, Oxygen-regulated protein 1, CFA54, Alpha-parvin, CAP2, MYH10, Ephrin-B2, Ephrin-B, Dynein 2, cytoplasmic, heavy chain, NPX1, PKA-reg (cAMPdependent), CFA74, SPAG16, Alpha-actinin, RFX3, SLUG, MENA, Ephrin-A receptors, SHANK, TMEM100, KIAA1377(HIP5), CLR3, Notch, Galpha(q)-specific peptide GPCRs, CYFIP1, HYDIN, Rab-3A, TSGA2, ATF/CREB, MAP-1B, CCDC176, DNAI1, DZIP, Dynein, axonemal, intermediate chains, NME5, SNAP-29, LRGUK, ILPIP, KAD7, C2orf39, RSPH4A, SNX10 $297 \quad 4.07 \mathrm{E}-13 \quad 1.77 \mathrm{E}-10 \quad 36$

KIF21A, DNAH5, CCDC39, MEK1(MAP2K1),

ARMC4, DNAH1, Hap-1, SPAG17, Dynein, axonemal, light chains, HIF1A, MEK1/2, DNAH9, MIP-T3, LRRC50, DNAH6, Dynein, axonemal, heavy chains, CFA54, Dynein 2, cytoplasmic, heavy chain, SPAG16, DNAH11, RFX3, Sp17, KIF3B, Huntingtin, HYDIN, DNAH3, MAP1B, DNAH2, DNAI1, Dynein, axonemal, intermediate chains, NME5, 


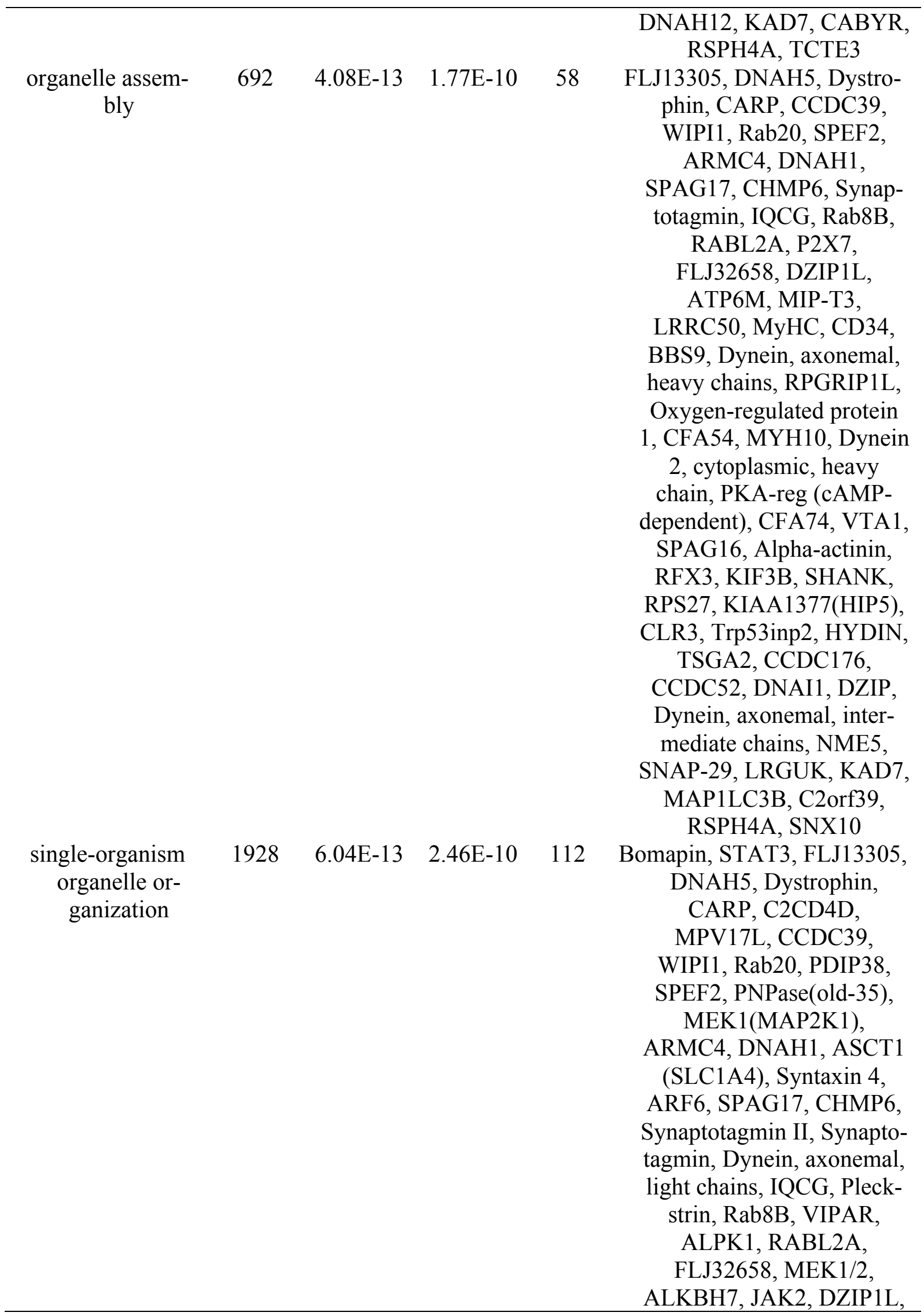


movement of cell or subcellular component
ATP6M, Rab-3, MIP-T3, MNS1, LRRC50, MyHC, Carbohydrate sulfotransferases, YSK4, BBS9, WHDC1, WRCH-1, Dynein, axonemal, heavy chains, RPGRIP1L, Oxygen-regulated protein 1 , CFA54, Alpha-parvin, ENO4, CAP2, MYH10, Profilin II, MRE11, Dynein 2, cytoplasmic, heavy chain, PKA-reg (cAMPdependent), CFA74,

SPAG16, Alpha-actinin, RFX3, MENA, KIF3B, Bim, SHANK, RPS27, GMIP, NMT1,

KIAA1377(HIP5), GRO-1, SPATA18, CLR3, Galpha(q)-specific peptide GPCRs, Huntingtin, Profilin, HYDIN, Rab-3A, TSGA2, FGF10, ATF/CREB, EPS8, MAP1B, CCDC176, CCDC52, DNAI1, DZIP, FGD3, Nmyristoyltransferase,

PI4KII, Dynein, axonemal, intermediate chains,

NUAK2, FAT1, ANKFY1, NME5, SNAP-29, Phosphatase regulator (inhibitor), Syntenin 1, LRGUK, VAMP4, NCK1, Beta adducin, KAD7, BET1, Nephrocystin-4, ABI2, MAP1LC3B, C2orf39, RSPH4A, Alphainternexin, SNX10

$1795 \quad 1.04 \mathrm{E}-12 \quad 3.99 \mathrm{E}-10 \quad 106$ beta-MHC, KIF21A, CD84, STAT3, DNAH5, Dystrophin, CCDC39, MEK1(MAP2K1), ARMC4, SLIT3, DNAH1, PVR, PLET1, LYST, Hap1, MGF, Olfactory receptor, 
anatomical structure formation involved in
ARF6, LTBR1, SPAG17, Vinculin, DHSO, Dynein, axonemal, light chains, PDE, HIF1A, RABL2A, FAT3, HES1, MEK1/2, B3GN1, ROBO1, DNAH9, JAK2, Islet-1, Galpha(q)specific nucleotide-like

GPCRs, MIP-T3, LRRC50, Ephrin-A receptor 5, DNAH6, MyHC, CD34, Carbohydrate sulfotransferases, Dynein, axonemal, heavy chains, CFA54, A1pha-parvin, ENO4, MYH10, Lyn, Ephrin-B2, Ephrin-B, Dynein 2, cytoplasmic, heavy chain, STRBP, CAPZA1, SPAG16, Alpha-actinin, DNAH11, GRO-3, RFX3, SLUG, MENA, Ephrin-A receptors, Sp17, KIF3B,

DAGK, CCR1, Hck, GRO1, SPP lyase, CAPZA, CLR3, Notch, SLAM, Galpha(q)-specific peptide GPCRs, Huntingtin, GFRalpha1, HYDIN, CCL27, FGF10, ATF/CREB, EPS8, C4orf31, DNAH3, MAP1B, CAPZA2, DNAH2, DNAI1, Alpha-actinin 3, HDL proteins, Dynein, axonemal, intermediate chains, FAT1, PLTP,

NME5, Syntenin 1, NCK1, RANK(TNFRSF11A), DNAH12, KAD7, Nephrocystin-4, ABI2, CABYR,

PI3K reg class IA, C2orf39, RSPH4A, CCNYL1, PSTPIP2, TCTE3

$1410 \quad 6.56 \mathrm{E}-12 \quad 2.39 \mathrm{E}-09 \quad 88$

FLJ13305, DNAH5, Dystrophin, CARP, IL-15, CCDC39, SF3B14a, 


\begin{tabular}{|c|c|c|c|c|c|}
\hline $\begin{array}{c}\text { epithelial cilium } \\
\text { movement }\end{array}$ & 29 & $1.03 \mathrm{E}-11$ & $3.55 \mathrm{E}-09$ & 12 & 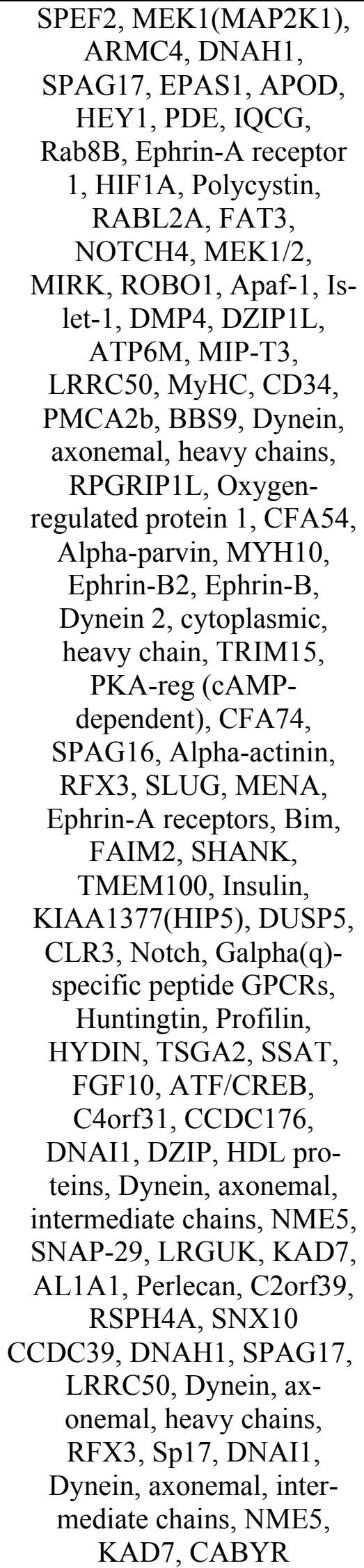 \\
\hline
\end{tabular}




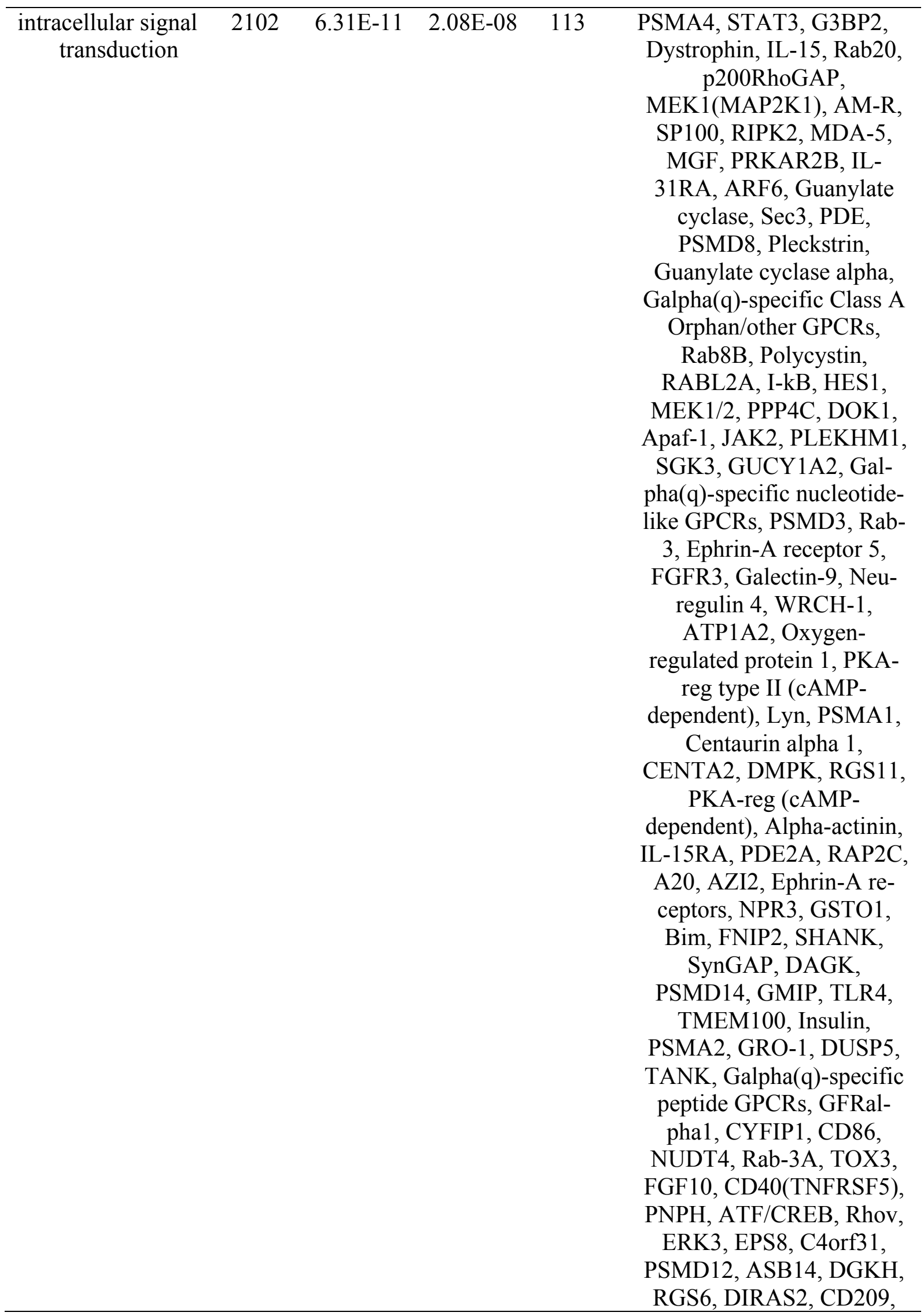




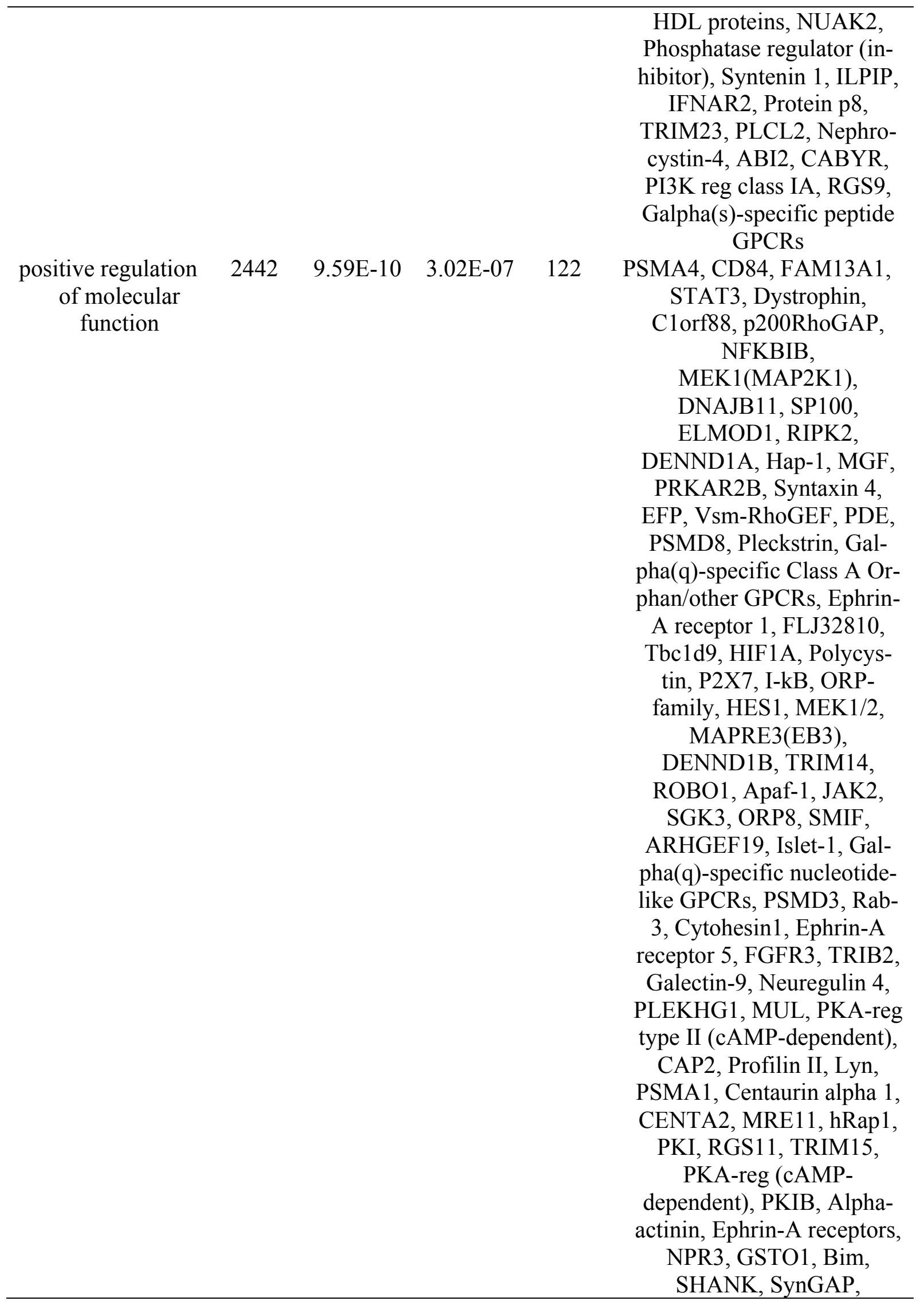


regulation of re-

4960

1.14E-09 3.42E-07

sponse to stimu-

lus
PSMD14, CREB3, GMIP,

TLR4, RGSS22, Insulin, Fibulin-1, Pyrin (MEFV), PSMA2, GRO-1, DUSP5, TANK, Galpha(q)-specific peptide GPCRs, Huntingtin, GFRalpha1, Profilin, Rab3A, NEK5, FGF10, CD40(TNFRSF5), ATF/CREB, FAM116B, PSMD12, SERINC5, GITRL, Glutaredoxin, FGD3, RGS6, DIRAS2, HDL proteins, Phosphatase regulator (inhibitor), ILPIP, NCK1, RANK(TNFRSF11A), Beta adducin, AL1A1, TRIM23, PLCL2, LULL1, PI3K reg class IA, RGS9, Galpha(s)specific peptide GPCRs, MAP1LC3B, NPNT, Glutaredoxin 1 Bomapin, beta-MHC, PSMA4, CD84, FAM13A1, STAT3, G3BP2, PODN, Dystrophin, CARP, MPV17L, IL-15, WFDC1, H-2L(d), p200RhoGAP, FLJ20898, OSF-2, REVERBalpha, NFKBIB, TRalpha, MHC Class I alpha chain, PNPase(old-35), MEK1(MAP2K1), C2, DOT1, SLIT3, SP100, PVR, RIPK2, MDA-5, DENND1A, Hap-1, MGF, SNX1, IL-31RA, Syntaxin 4, Trim30, ARF6, Guanylate cyclase, PRAM-1, APOD, EFP, HEY1, VsmRhoGEF, Synaptotagmin, Dynein, axonemal, light chains, PDE, TREML2, ACP33, PSMD8, Pleckstrin, Galpha(q)-specific Class A Orphan/other 
GPCRs, CD300LG, ZNF364, FLJ32810, ALPK1, HIF1A, Fibulin-5, Peregrin, P2X7, I-kB, ORPfamily, NOTCH4, HES1, MEK1/2, ATF-3, PPP4C, DENND1B, SAMHD1, DOK1, C3orf58, ROBO1, Apaf-1, JAK2, SGK3, ORP8, ARHGEF 19, Islet-1, DMP4, Galpha(q)-specific nucleotide-like GPCRs, PSMD3, MIP-T3, Cytohesin1, Ephrin-A receptor 5, FGFR3, MyHC, TRIB2, CD34, Galectin-9, Carbohydrate sulfotransferases, Neuregulin 4, Ceb1, TRAFD1, PLEKHG1, WRCH-1, Krm1, RPGRIP1L, C4B protein, Lyn, PSMA1, HLA-E, Ephrin-B2, DMPK, MRE11, Ephrin-B, Dynein

2, cytoplasmic, heavy chain, hRap1, CysLT2 receptor, RGS11, TRIM15, HA12 (rodent), Alphaactinin, GRO-3, TOP3A, SLUG, MENA, PDE2A, Coagulation factor $X$, Orexin receptor 1, A20, EphrinA receptors, GSTO1, Bim, FAIM2, SCAI, SHANK, SynGAP, Fibrinogen gamma, DAGK, PSMD14, IL-1 alpha, CREB3, CCR1, Hck, GMIP, TLR4, C4, RGSS22, NMT1, TMEM100, Insulin, Fibulin-1, Pyrin (MEFV), PSMA2, ADAR1, GRO-1, DUSP5, CD276, TANK, BTNL2, Asporin, Notch, Orexin receptor, SLAM, Galpha(q)-specific peptide GPCRs, Huntingtin, 
microtubule-based process
CYFIP1, HA11, CD86, CCL27, FGF10, CD40(TNFRSF5), PNPH, ATF/CREB, Rhov, RNF34, EPS8, PSMD12, CQ087, GITRL, Glutaredoxin, HLA-C, PEAR1, FGD3, PCPH, N-

myristoyltransferase, PIP1, RGS6, DIRAS2, HLA-B, Alpha-actinin 3, CD209, HDL proteins, BCAP, NME5, Phosphatase regulator (inhibitor), Syntenin 1, MPP1, PI3K reg class IA (p55-gamma), ILPIP, NCK1, RANK(TNFRSF11A), USP18, IFNAR2, GREM2, Thyroid hormone receptor, PLCL2, Nephrocystin-4, PI3K reg class IA, RGS9, CD300LF, IPP-2, Galpha(s)-specific peptide GPCRs, DUSP18, TIG2, DDX60, NPNT,

SERPINB9, Glutaredoxin 1 $696 \quad 3.79 \mathrm{E}-09 \quad 1.09 \mathrm{E}-06 \quad 50$ KIF21A, DNAH5, CCDC39, SPEF2, MEK1(MAP2K1), ARMC4, DNAH1, LYST, Hap-1, SPAG17, Dynein, axonemal, light chains,

IQCG, HIF1A, FLJ32658, MEK1/2, DNAH9, MIPT3, LRRC50, DNAH6, MyHC, Dynein, axonemal, heavy chains, Oxygenregulated protein 1, CFA54, Dynein 2, cytoplasmic, heavy chain, CFA74, SPAG16, DNAH11, RFX3, Sp17, KIF3B,

KIAA1377(HIP5), Huntingtin, HYDIN, TSGA2, FGF10, DNAH3, MAP-1B, DNAH2, CCDC52, DNAI1, Dynein, axonemal, 


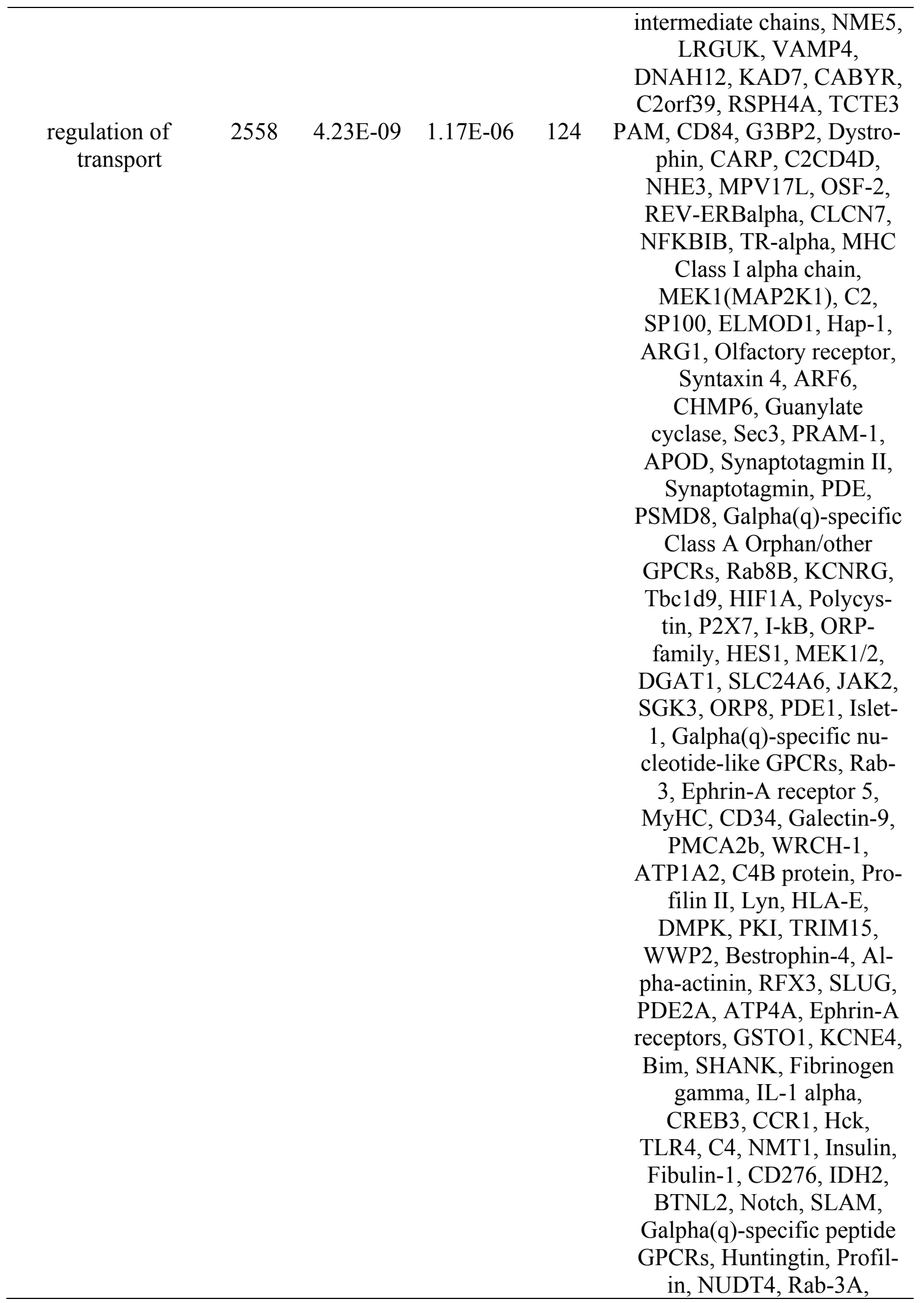


phosphorus meta- $2600 \quad 5.92 \mathrm{E}-09 \quad 1.54 \mathrm{E}-06 \quad 125$ bolic process

\section{FGF10, CD40(TNFRSF5), ATF/CREB, DNAJC13, SCN3A, MAP-1B, Glu- taredoxin, $\mathrm{N}-$}

myristoyltransferase, HLAB, CHD7, HDL proteins, PLTP, ANKFY1, Phosphatase regulator (inhibitor), Syntenin 1, UGCG, PI3K

reg class IA (p55-gamma), PARP-10, VAMP4, RANK(TNFRSF11A),

Thyroid hormone receptor, PI3K reg class IA, Galpha(s)-specific peptide GPCRs, TIG2, MAP1LC3B, Glutaredoxin 1

beta-MHC, RPS6KL1, PSMA4, STAT3, GNPTA, CTP synthase, IL-15, DDHD1,

MEK1(MAP2K1), PLB1, PHEX, Stk33, RIPK2, KIAA1274, LYST, MGF, PRKAR2B, IL-31RA, Mdh1b, Guanylate cyclase, Glycogen phosphorylase, CDP-diacylglycerol synthase, DHSO, PDE, PSMD8, Pleckstrin,

Guanylate cyclase alpha, Ephrin-A receptor 1, ALPK1, Polycystin, P2X7, MEK1/2, PPP4C, MIRK, SAMHD1, DOK1, JAK2, SGK3, GUCY1A2, PDE1, DMP4, Galpha(q)-specific nucleotide-like GPCRs, PSMD3, Ephrin-A receptor 5, FGFR3, IDH1, MyHC, TRIB2, Galectin-9, PMCA2b, Neuregulin 4, YSK4, PGS1, ATP1A2, NNTM, PKA-reg type II (cAMP-dependent), ENO4, ATP6V1B2, MANA, Lyn, 
regulation of cell 4033 5.99E-09 1.54E-06

communication
PSMA1, DMPK, PKI, CDS2, PKA-reg (cAMPdependent), PKIB, Alphaactinin, PDE2A, Ephrin-A receptors, NPR3, AKD1, FNIP2, SHANK, SynGAP, DAGK, PSMD14, Hck, TLR4, Insulin, PBEF, PSMA2, DUSP5, NT5C3, IDH2, Galpha(q)-specific peptide GPCRs, GFRalpha1, CD86, NUDT4, FLAD1, NEK5, MOK, FGF10, PNPH, ATF/CREB, ERK3, PSMD12, SERINC5, ADSSL1, LDHB, MTHFD2L, ATP6V1B, PDE1B, DGKH, PCPH, PI4KII, AGPHD1, TXL-2, HDL proteins, PPP1R3B, BCAP, NUAK2, C9orf98, NME5, Phosphatase regulator (inhibitor), MPP1,

LRGUK, PI3K reg class IA (p55-gamma), ILPIP, NCK1, KAD7, AL1A1, $\mathrm{ABI} 2, \mathrm{PI} 3 \mathrm{~K}$ reg class IA, DUSP18, FRK

PSMA4, CD84, FAM13A1, STAT3, G3BP2, PODN, Dystrophin, CARP, MPV17L, IL-15, p200RhoGAP, FLJ20898, OSF-2, REV-ERBalpha, NFKBIB, TR-alpha, MEK1(MAP2K1), DOT1, SLIT3, SP100, RIPK2, DENND1A, Hap-1, MGF, SNX1, IL-31RA, Syntaxin 4, Trim30, ARF6, Guanylate cyclase, APOD, EFP, HEY1, Vsm-RhoGEF, Synaptotagmin, Dynein, axonemal, light chains, PDE, PSMD8, Pleckstrin, Galpha(q)-specific Class A 
Orphan/other GPCRs, Rab8B, ZNF364, FLJ32810, HIF1A, Peregrin, P2X7, I-kB, ORPfamily, NOTCH4, HES1, MEK1/2, ATF-3, DOK1, C3orf58, SLC24A6, ROBO1, Apaf-1, JAK2, SGK3, ORP8, ARHGEF19, PDE1, Islet-1, DMP4, Galpha(q)-specific nucleotidelike GPCRs, PSMD3, Rab3, MIP-T3, Cytohesin1, Ephrin-A receptor 5, FGFR3, TRIB2, Galectin-9, PMCA2b, Neuregulin 4, PLEKHG1, WRCH-1, Krm1, ATP1A2, RPGRIP1L, Profilin II, Lyn, PSMA1, DMPK, MRE11, Dynein 2, cytoplasmic, heavy chain, hRap1, CysLT2 receptor, RGS11, TRIM15, Alphaactinin, TOP3A, RFX3, SLUG, Coagulation factor $\mathrm{X}$, Orexin receptor 1, A20, Ephrin-A receptors, GSTO1, Bim, FAIM2, SCAI, SHANK, SynGAP, Fibrinogen gamma, DAGK, PSMD14, IL-1 alpha, CREB3, CCR1, GMIP, TLR4, RGSS22, NMT1, TMEM100, Insulin, Fibulin-1, PSMA2, ADAR1, DUSP5, TANK, BTNL2, Asporin, Notch, Orexin receptor, SLAM, Galpha(q)specific peptide GPCRs, Huntingtin, CD86, Profilin, Rab-3A, FGF10, CD40(TNFRSF5),

ATF/CREB, Rhov, RNF34, EPS8, MAP-1B, PSMD12, CQ087, GITRL,

Calsyntenin-2, Glutaredox- 


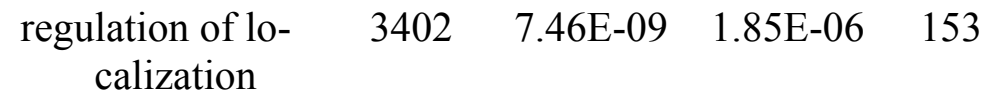

in, PEAR1, SHANK2, FGD3, PCPH, Nmyristoyltransferase, PIP1, RGS6, DIRAS2, CHD7, Alpha-actinin 3, HDL proteins, BCAP, NME5, Phosphatase regulator (inhibitor), Syntenin 1, ILPIP, NCK1, RANK(TNFRSF11A), USP18, IFNAR2, GREM2, Thyroid hormone receptor, PLCL2, Nephrocystin-4, PI3K reg class IA, RGS9, IPP-2, Galpha(s)-specific peptide GPCRs, DUSP18, DDX60, NPNT, Glutaredoxin 1

PAM, CD84, STAT3, G3BP2, PODN, Dystrophin, CARP, C2CD4D, NHE3, MPV17L, CCDC39, OSF-2, REVERBalpha, CLCN7, NFKBIB, TR-alpha, MHC Class I alpha chain, MEK1(MAP2K1), C2, ARMC4, SP100, PLET1, ELMOD1, Hap-1, MGF, ARG1, Olfactory receptor, Syntaxin 4, ARF6, CHMP6, Guanylate cyclase, Sec3, PRAM-1, Vinculin, APOD, Synaptotagmin II, Synaptotagmin, PDE, PSMD8, Galpha(q)specific Class A Orphan/other GPCRs, Rab8B, Ephrin-A receptor 1, KCNRG, Tbc1d9, HIF1A, Polycystin, LDB2, P2X7, IkB, ORP-family, NOTCH4, HES1, MEK1/2, DGAT1, ALKBH7, SLC24A6, ROBO1, JAK2, SGK3, ORP8, PDE1, Islet-1, Galpha(q)-specific nucleotide- 
like GPCRs, Rab-3, LRRC50, Ephrin-A receptor 5, MyHC, CD34, Galectin-9, PMCA2b, Carbohydrate sulfotransferases, Neuregulin 4, WRCH-1, ATP1A2, Dynein, axonemal, heavy chains, C4B protein, Profilin II, Lyn, HLA-E, DMPK, PKI, TRIM15, WWP2, Bestrophin-4, Alpha-actinin, DNAH11, GRO-3, RFX3, SLUG, PDE2A, RAP2C, ATP4A, Coagulation factor $\mathrm{X}$, Ephrin-A receptors, GSTO1, KCNE4, Bim, SCAI, SHANK, Fibrinogen gamma, IL-1 alpha, CREB3, CCR1, Hck, TLR4, PLEKHM2, C4, NMT1, Insulin, Fibulin-1, GRO-1, CD276, IDH2, BTNL2, CLR3, Notch, SLAM, Galpha(q)-specific peptide GPCRs, Huntingtin, Trp53inp2, Profilin, NUDT4, Rab-3A, CCL27, FGF10, CD40(TNFRSF5), ATF/CREB, DNAJC13, SCN3A, MAP-1B, GITRL,

Glutaredoxin, Nmyristoyltransferase, HLAB, CHD7, HDL proteins, PLTP, ANKFY1, Phosphatase regulator (inhibitor), Syntenin 1, MPP1, UGCG, PI3K reg class IA ( $\mathrm{p} 55-$ gamma), PARP-10, VAMP4, NCK1, RANK(TNFRSF11A),

Thyroid hormone receptor, PI3K reg class IA, Galpha(s)-specific peptide GPCRs, TIG2, MAP1LC3B, Glutaredoxin 
XMWAS: $R$ package for data integration and network analysis




NFKBIB, TR-alpha, MEK1(MAP2K1), DOT1, SLIT3, SP100, RIPK2, DENND1A, Hap-1, MGF, SNX1, IL-31RA, Syntaxin 4, Trim30, ARF6, Guanylate cyclase, APOD, EFP, HEY1, Vsm-RhoGEF, Synaptotagmin, Dynein, axonemal, light chains, PDE, PSMD8, Pleckstrin, Galpha(q)-specific Class A Orphan/other GPCRs, Rab8B, ZNF364, FLJ32810, HIF1A, Peregrin, P2X7, I-kB, ORPfamily, NOTCH4, HES1, MEK1/2, ATF-3, DOK1, C3orf58, SLC24A6, ROBO1, Apaf-1, JAK2, SGK3, ORP8, ARHGEF19, PDE1, Islet-1, DMP4, Galpha(q)-specific nucleotidelike GPCRs, PSMD3, Rab3, MIP-T3, Cytohesin1, Ephrin-A receptor 5, FGFR3, TRIB2, Galectin-9, Ca-ATPase 3, PMCA2b, Neuregulin 4, PLEKHG1, WRCH-1, Krm1, ATP1A2, RPGRIP1L, Profilin II, Lyn, PSMA1, DMPK,

MRE11, Dynein 2, cytoplasmic, heavy chain, hRap1, CysLT2 receptor, RGS11, TRIM15, Alphaactinin, TOP3A, RFX3,

SLUG, Coagulation factor

$\mathrm{X}$, Orexin receptor 1, A20, Ephrin-A receptors, GSTO1, Bim, FAIM2, SCAI, SHANK, SynGAP, Fibrinogen gamma, DAGK, PSMD14, IL-1 alpha, CREB3, CCR1, GMIP, TLR4, RGSS22, NMT1, TMEM100, Insulin, Fibu- 
phosphate-

containing

compound met-

abolic process lin-1, PSMA2, ADAR1, DUSP5, TANK, BTNL2, Asporin, Notch, Orexin receptor, SLAM, Galpha(q)specific peptide GPCRs, Huntingtin, CD86, Profilin, Rab-3A, FGF10, CD40(TNFRSF5),

ATF/CREB, Rhov, RNF34, EPS8, MAP-1B, PSMD12, CQ087, GITRL, Calsyntenin-2, Glutaredoxin, PEAR1, SHANK2, FGD3, PCPH, Nmyristoyltransferase, PIP1, RGS6, DIRAS2, CHD7, Alpha-actinin 3, HDL proteins, BCAP, NME5, Phosphatase regulator (inhibitor), Syntenin 1, ILPIP, NCK1, RANK(TNFRSF11A), USP18, IFNAR2, GREM2, Thyroid hormone receptor, PLCL2, Nephrocystin-4, PI3K reg class IA, RGS9, IPP-2, Galpha(s)-specific peptide GPCRs, DUSP18,

DDX60, NPNT, Glutaredoxin 1 $2595 \quad 1.85 \mathrm{E}-08 \quad 3.97 \mathrm{E}-06 \quad 123$ beta-MHC, RPS6KL1, PSMA4, STAT3, GNPTA, CTP synthase, IL-15, MEK1(MAP2K1), PLB1, Stk33, RIPK2, KIAA1274, LYST, MGF, PRKAR2B, IL-31RA, Mdh1b, Guanylate cyclase, Glycogen phosphorylase, CDPdiacylglycerol synthase, DHSO, PDE, PSMD8,

Pleckstrin, Guanylate cyclase alpha, Ephrin-A receptor 1, ALPK1, Polycystin, P2X7, MEK1/2, PPP4C, MIRK, SAMHD1, DOK1, JAK2, SGK3, 
regulation of molecular function
GUCY1A2, PDE1, DMP4,

Galpha(q)-specific nucleotide-like GPCRs, PSMD3, Ephrin-A receptor 5, FGFR3, IDH1, MyHC, TRIB2, Galectin-9, PMCA2b, Neuregulin 4, YSK4, PGS1, ATP1A2, NNTM, PKA-reg type II (cAMP-dependent), ENO4, ATP6V1B2, MANA, Lyn, PSMA1, DMPK, PKI, CDS2, PKA-reg (cAMPdependent), PKIB, Alphaactinin, PDE2A, Ephrin-A receptors, NPR3, AKD1, FNIP2, SHANK, SynGAP, DAGK, PSMD14, Hck, TLR4, Insulin, PBEF, PSMA2, DUSP5, NT5C3, IDH2, Galpha(q)-specific peptide GPCRs, GFRalpha1, CD86, NUDT4, FLAD1, NEK5, MOK, FGF10, PNPH, ATF/CREB, ERK3, PSMD12, SERINC5, ADSSL1, LDHB, MTHFD2L, ATP6V1B, PDE1B, DGKH, PCPH, PI4KII, AGPHD1, TXL-2, HDL proteins, PPP1R3B, BCAP, NUAK2, C9orf98,

NME5, Phosphatase regulator (inhibitor), MPP1, LRGUK, PI3K reg class IA (p55-gamma), ILPIP, NCK1, KAD7, AL1A1, $\mathrm{ABI} 2, \mathrm{PI} 3 \mathrm{~K}$ reg class IA, DUSP18, FRK $3799 \quad 1.86 \mathrm{E}-08 \quad 3.97 \mathrm{E}-06 \quad 165$ TR-alpha, 


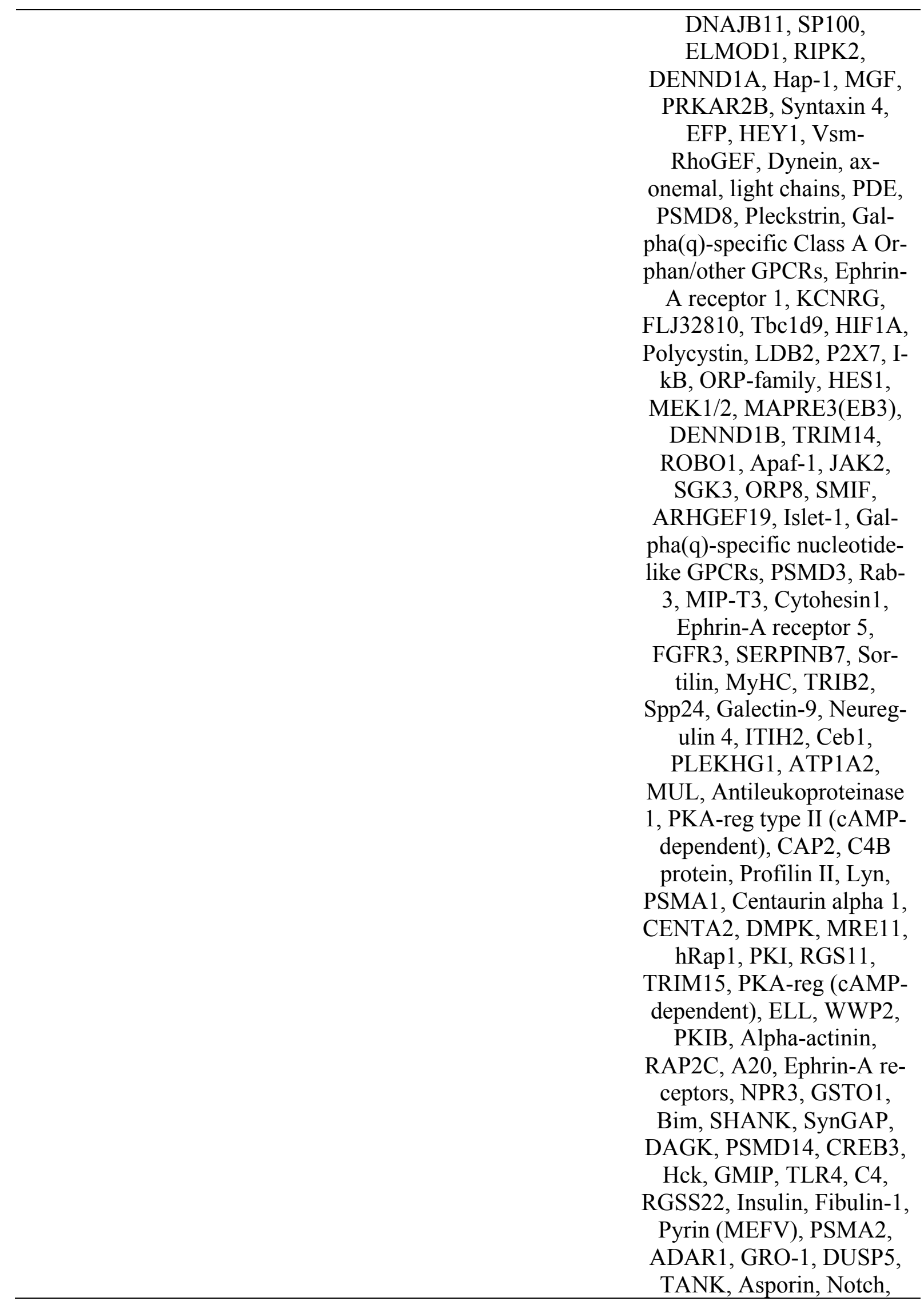


regulation of signal transduction
$3625 \quad 1.93 \mathrm{E}-08 \quad 3.97 \mathrm{E}-06 \quad 159$
SLAM, Galpha(q)-specific peptide GPCRs, Huntingtin, GFRalpha1, Mitd1, Profilin, Rab-3A, NEK5, FGF10, CD40(TNFRSF5), ATF/CREB, FAM116B, RNF34, PSMD12, SERINC5, GITRL, Glutaredoxin, AGAL, FGD3, RGS6, DIRAS2, HDL proteins, PPP1R3B, Phosphatase regulator (inhibitor), PI3K reg class IA ( $\mathrm{p} 55-$ gamma), PARP-10, ILPIP, NCK1, RANK(TNFRSF11A), Beta adducin, GREM2, AL1A1, Thyroid hormone receptor, TRIM23, PLCL2, LULL1, PI3K reg class IA, RGS9, IPP-2, Galpha(s)-specific peptide GPCRs, DUSP18, MAP1LC3B, CCNYL1, NPNT, SERPINB9, Glutaredoxin 1

PSMA4, CD84, FAM13A1, STAT3, G3BP2, PODN, Dystrophin, CARP, MPV17L, IL-15, p200RhoGAP, FLJ20898, OSF-2, REV-ERBalpha,

NFKBIB, TR-alpha, MEK1(MAP2K1), DOT1, SLIT3, SP100, RIPK2, DENND1A, Hap-1, MGF, SNX1, IL-31RA, Syntaxin 4, Trim30, ARF6, Guanylate cyclase, APOD, EFP, HEY1, Vsm-RhoGEF, Dynein, axonemal, light chains, PDE, PSMD8, Pleckstrin, Galpha(q)specific Class A Orphan/other GPCRs, ZNF364, FLJ32810, HIF1A, Peregrin, P2X7, IkB, ORP-family, NOTCH4, 
HES1, MEK1/2, ATF-3, DOK1, C3orf58, ROBO1, Apaf-1, JAK2, SGK3, ORP8, ARHGEF19, Islet-1, DMP4, Galpha(q)-specific nucleotide-like GPCRs, PSMD3, MIP-T3, Cytohe$\sin 1$, FGFR3, TRIB2, Galectin-9, Neuregulin 4, PLEKHG1, WRCH-1, Krm1, RPGRIP1L, Lyn, PSMA1, DMPK, MRE11, Dynein 2, cytoplasmic, heavy chain, hRap1, CysLT2 receptor, RGS11, TRIM15, Alpha-actinin, TOP3A, SLUG, Coagulation factor $\mathrm{X}$, Orexin receptor 1, A20, Ephrin-A receptors, GSTO1, Bim, FAIM2, SCAI, SHANK, SynGAP, Fibrinogen gamma, DAGK, PSMD14, IL-1 alpha, CREB3, CCR1, GMIP, TLR4, RGSS22, NMT1, TMEM100, Insulin, Fibulin-1, PSMA2, ADAR1, DUSP5, TANK, BTNL2, Asporin, Notch, Orexin receptor, SLAM, Galpha(q)specific peptide GPCRs, Huntingtin, CD86, FGF10, CD40(TNFRSF5),

ATF/CREB, Rhov, RNF34, EPS8, PSMD12, CQ087, GITRL, Glutaredoxin, PEAR1, FGD3, PCPH, Nmyristoyltransferase, PIP1, RGS6, DIRAS2, Alphaactinin 3, HDL proteins, BCAP, NME5, Phosphatase regulator (inhibitor), Syntenin 1, ILPIP, NCK1, RANK(TNFRSF11A), USP18, IFNAR2, GREM2, Thyroid hormone receptor, PLCL2, Nephrocystin-4, 


$\begin{array}{cccc}\substack{\text { immune system } \\ \text { process }} & 3048 \quad 1.95 \mathrm{E}-08 & 3.97 \mathrm{E}-06 & 139\end{array}$

PI3K reg class IA, RGS9, IPP-2, Galpha(s)-specific peptide GPCRs, DUSP18, DDX60, NPNT, Glutaredoxin 1

PSMA4, CD84, CTP synthase, IL-15, H-2L(d), Mca32, SPEF2, TR-alpha, MHC Class I alpha chain, MEK1(MAP2K1), C2, SP100, RIPK2, MDA-5, LYST, MGF, IL-31RA, LTBR1, EPAS1, EFP, PDE, TREML2, ACP33, PSMD8, Pleckstrin, Galpha(q)-specific Class A Orphan/other GPCRs, Rab8B, CD300LG, HIF1A, SUSD2, ADAMDEC1, P2X7, I-kB, ORP-family, NOTCH4, HES1, MEK1/2, DENND1B, SAMHD1, TRIM14, JAK2, MR1, PDE1, DMP4, Galpha(q)specific nucleotide-like GPCRs, PSMD3, FLVCR, Rab-3, MxA, Ku70, MyHC, CD34, Galectin-9, Carbohydrate sulfotransferases, Ceb1, Antileukoproteinase 1, TFE3, C4B protein, Lyn, PSMA1, HLA-E, Ephrin-B2, Ephrin-B, Dynein 2, cytoplasmic, heavy chain, CysLT2 receptor, TRIM15, CAPZA1, HA12 (rodent), Alphaactinin, GRO-3, MENA, PDE2A, A20, OASL, AZI2, Ephrin-A receptors, KIF3B, Bim, Fibrinogen gamma, PSMD14, IL-1 alpha, CCR1, CCR9, Hck, TLR4, C4, Insulin, Pyrin (MEFV), PSMA2, ADAR1, GRO-1, CD276, SPP lyase, TANK, Galectin-8, 
single-organism

14419

cellular process
CAPZA, Notch, SLAM, Galpha(q)-specific peptide GPCRs, ISG20, CYFIP1, HA11, CD86, CCL27, TAPBPL, FGF10, CD40(TNFRSF5), PNPH, ATF/CREB, EPS8, PSMD12, SERINC5, ADSSL1, CAPZA2, GITRL, HLA-C, PDE1B, HLA-B, Artemis, CHD7, PI4KII, CD209, HDL proteins, BCAP, OSTM1 (grey-lethal), NCK1, RANK(TNFRSF11A), Beta adducin, IFNAR2, KAD7, Thyroid hormone receptor, PLCL2, RPESP, PI3K reg class IA, CD300LF,

DDX60, FRK, SERPINB9, SNX10

Bomapin, beta-MHC, RPS6KL1, PAM, KIF21A, PSMA4, CD84, FAM13A1, B4GT4, STAT3, G3BP2, FLJ13305, PODN, DNAH5, Dystrophin, GNPTA, CARP, CTP synthase, C2CD4D, NHE3, MPV17L, IL-15, OLFML2A, C1orf88, CCDC39, H-2L(d), Mca32, WIPI1, Trehalase, DPYD, Rab20, PDIP38, p200RhoGAP, SPEF2, FLJ20898, OSF-2, REV-

ERBalpha, HDAC11, NFKBIB, STOML3, ZNF281, TR-alpha, MHC Class I alpha chain, PNPase(old-35), GPR141, MEK1(MAP2K1), C2, ARMC4, DOT1, SLIT3, PLB1, AM-R, DNAH1, PHEX, DNAJB11, SNX2, SP100, PVR, PLET1, RIPK2, MDA-5, ASCT1 
(SLC1A4), ZNF313, LYST, Hap-1, MGF, SNX1, PRKAR2B, ARG1, IL-31RA, Olfactory receptor, Syntaxin 4, ARF6, LTBR1, SPAG17, SCPEP1, CHMP6, Mdh1b, Guanylate cyclase, EPAS1, DMKN, Sec3, PRAM-1, Vinculin, APOD, EFP, Glycogen phosphorylase, HEY1, CEP78, Synaptotagmin II, VE-cadherin, NCKX3, Synaptotagmin, CD-MPR, CDPdiacylglycerol synthase, DHSO, HIST3H2A,

Dynein, axonemal, light chains, PDE, IQCG, TREML2, MGAT4A, ACP33, KCNQ1OT1, PSMD8, Pleckstrin,

LRP12, Guanylate cyclase alpha, Galpha(q)-specific Class A Orphan/other GPCRs, Rab8B, HPLC2, Ephrin-A receptor 1, VIPAR, FLJ32810, ALPK1, HIF1A, Polycystin, Fibulin-5, Tmem195, RABL2A, FAT3, P2X7, IkB, ORP-family, NOTCH4, HES1, FLJ32658, Vitrin, MEK1/2, DGAT1, ATF-3, Olr1598, PPP4C, MIRK, OLAH, MAPRE3(EB3), DENND1B, SAMHD1, VN1R4, B3GN1, DOK1, C3orf58, Egflam, ALKBH7, SLC24A6, ROBO1, DNAH9, Apaf-1, HAT1, JAK2, PLEKHM1, MR1, SGK3, ORP8, SMIF, GUCY1A2, ARHGEF19, ZCCHC14, PDE1, Islet-1, DMP4, Galpha(q)-specific nucleotide-like GPCRs, 
WDR93, PSMD3, FLVCR, DZIP1L, ATP6M, Rab-3, MIP-T3, Cytohesin1, MNS1, LRRC50, Ephrin-A receptor 5, MxA, FGFR3, FANCM, DNAH6, DYDC1, IDH1, RETSAT, $\mathrm{Ku} 70$, Sortilin, MyHC, VATPase E subunit, OGFR, CD34, Spp24, Galectin-9, Ca-ATPase3, PCDHB8, NHLRC2, PMCA2b, Carbohydrate sulfotransferases, Neuregulin 4, Ceb1, Scd2, YSK4, BBS9, PGS1, TRAP25, WHDC1, WRCH-1, Krm1, ATP1A2, Dynein, axonemal, heavy chains, RPGRIP1L, Oxygen-regulated protein 1 , NNTM, CFA54, Alphaparvin, PKA-reg type II (cAMP-dependent), ENO4, CAP2, MYH10, ATP6V1B2, VANGL1, MANA, Profilin II, Lyn, PSMA1, Centaurin alpha 1, CENTA2, HLA-E, EphrinB2, DMPK, PRIC285,

MRE11, Ephrin-B, Dynein 2, cytoplasmic, heavy chain, PYGM, LMLN, NPX1, STRBP, CNDP2, PKI, CysLT2 receptor, RGS11, CDS2, TRIM15, G-protein alpha-14, CAPZA1, PKA-reg (cAMP-dependent), HA12 (rodent), CFA74, KIAA1340, VTA1, SPAG16, Alpha-actinin, cMaf, DNAH11, IL-15RA, GRO-3, TOP3A, RFX3, SLUG, MENA, PDE2A, RAP2C, ATP4A, Orexin receptor 1, A20, OASL, AZI2, ABBA, Ephrin-A re- 
ceptors, GALRL, NPR3, GSTO1, Sp17, AKD1, KCNE4, KIF3B, Bim, SLC4A11, FAIM2, FNIP2,

SHANK, SynGAP, Fibrinogen gamma, DAGK, PSMD14, IL-1 alpha, CREB3, CCR1, CCR9, RPS27, TFIIF, beta subunit, Hck, GMIP, TLR4, NAR4, SCO2, NMT1, TMEM100, Insulin, Fibulin-1, PBEF, PSMA2, GNT-IV, Ces1 (mouse), ADAR1, KIAA1377(HIP5), GRO-1, DUSP5, CD276, SPP lyase, TANK, Galectin-8, NT5C3, PADI6, IDH2, SPATA18, CAPZA, CLR3, Asporin, FLJ46266, Notch, Orexin receptor, SLAM, APBA2BP, MCFD2, Galpha(q)-specific peptide

GPCRs, ISG20, Huntingtin, GFRalpha1, CYFIP1, HA11, CD86, Mitd1, POLR2G, Trp53inp2, Profilin, NUDT4, HYDIN, Rab-3A, CCL27, TOX3, TSGA2, ACADM, CBR1, FLAD1, ES130 (p180), MOK, FGF10, CD40(TNFRSF5), OR52N5, PNPH, T6441, ATF/CREB, Rhov, RNF34, ERK3, DNAJC13, EPS8, C4orf31, SCN3A, DNAH3, MAP-1B, PSMD12, SERINC5, ADSSL1, CQ087, LDHB, CAPZA2, FADS2, MTHFD2L, GITRL, CCDC176, Calsyntenin-2, SETDB2, ASB14, Glutaredoxin, DNAH2, MMSA, HLA-C, PEAR1, ATP6V1B, CCDC52, DNAI1, 
SHANK2, AGAL, DZIP, CGI-128, ZNF541, AL3A1, Heme oxygenase 2 , PDE1B, WDT2, FGD3, DGKH, PCPH, Nmyristoyltransferase,

PCDHB16, RGS6, GPR35, DIRAS2, HLA-B, ATP6V1E, Artemis, CHD7, Alpha-actinin 3, PI4KII, FBX6, AGPHD1, TXL-2, ANKRD28, CD209, HDL proteins, PPP1R3B, SIX5, BCAP, Dynein, axonemal, intermediate chains, NUAK2, C9orf98, FAT1, NRIF2, PLTP, ANKFY1, NME5, SNAP-29, Phosphatase regulator (inhibitor), OSTM1 (grey-lethal), Syntenin 1, ATP8B3, MPP1, UGCG, LRGUK, PI3K reg class IA ( $\mathrm{p} 55-$ gamma), PARP-10, VAMP4, ILPIP, NCK1, RANK(TNFRSF11A), P2Y4, NABP1, DNAH12, Beta adducin, IFNAR2, GREM2, KAD7, Protein p8, AL1A1, Thyroid hormone receptor, BET1, TRIM23, UOX, PLCL2, Nephrocystin-4, DIP13 beta, GSTT2, ACSS1, PTER, ABI2, TSNAXIP1, CABYR, LULL1, KIAA0317, PI3K reg class IA, RGS9, CD300LF, HPSE2, IPP-2, Galpha(s)specific peptide GPCRs, C8orf34, DUSP18, TIG2, MAP1LC3B, Perlecan, C2orf39, FAM188A, RSPH4A, Olfr554, CCNYL1, NPNT, Alphainternexin, MCEE, FRK, 


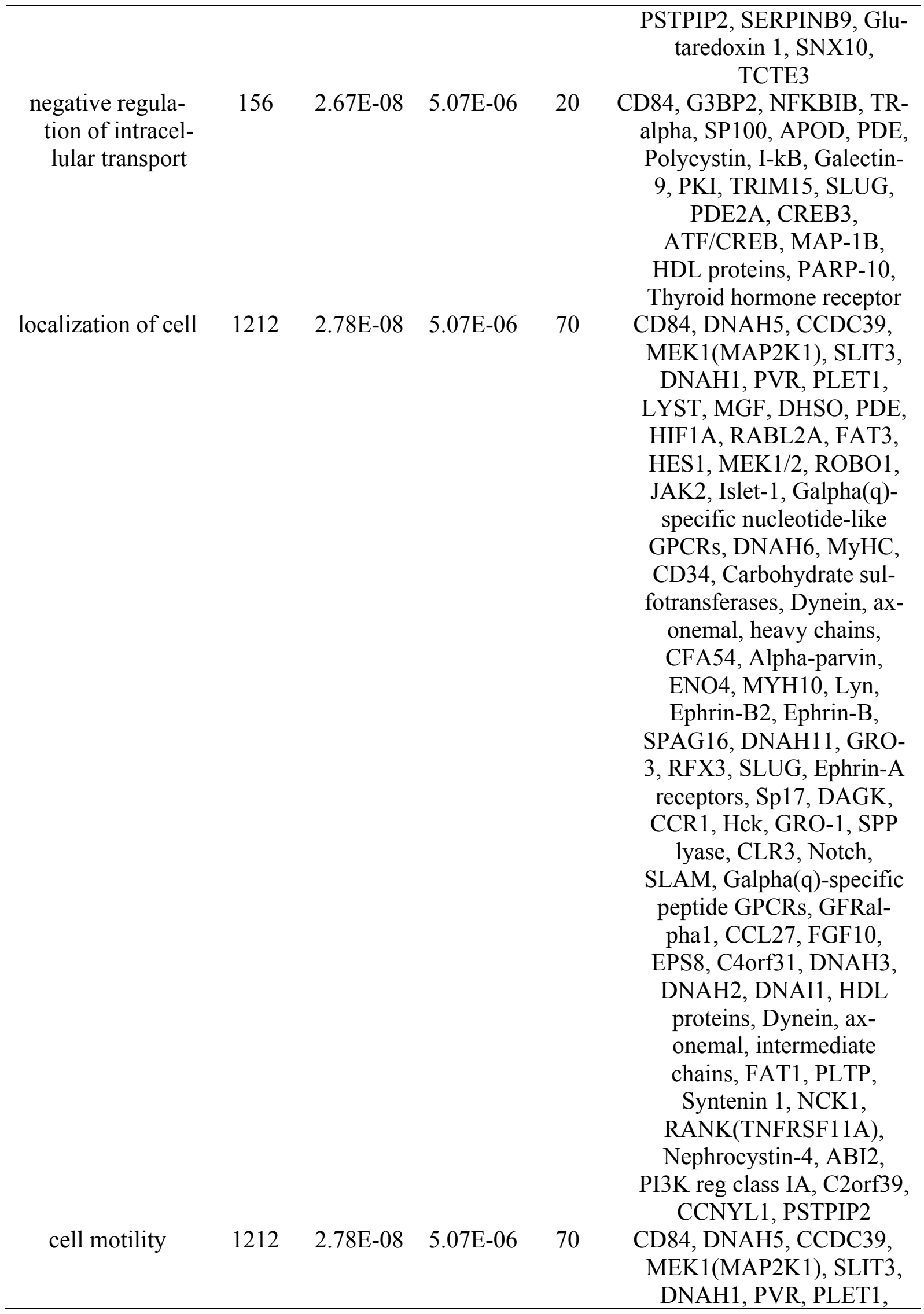


single-organism $16117 \quad 3.41 \mathrm{E}-08 \quad 6.05 \mathrm{E}-06$ process
LYST, MGF, DHSO, PDE, HIF1A, RABL2A, FAT3, HES1, MEK1/2, ROBO1, JAK2, Islet-1, Galpha(q)specific nucleotide-like GPCRs, DNAH6, MyHC, CD34, Carbohydrate sulfotransferases, Dynein, axonemal, heavy chains, CFA54, Alpha-parvin, ENO4, MYH10, Lyn, Ephrin-B2, Ephrin-B, SPAG16, DNAH11, GRO3, RFX3, SLUG, Ephrin-A receptors, Sp17, DAGK, CCR1, Hck, GRO-1, SPP lyase, CLR3, Notch, SLAM, Galpha(q)-specific peptide GPCRs, GFRalpha1, CCL27, FGF10, EPS8, C4orf31, DNAH3, DNAH2, DNAI1, HDL proteins, Dynein, axonemal, intermediate chains, FAT1, PLTP, Syntenin 1, NCK1, RANK(TNFRSF11A), Nephrocystin-4, ABI2, PI3K reg class IA, C2orf39, CCNYL1, PSTPIP2

Bomapin, beta-MHC, RPS6KL1, PAM, KIF21A, PSMA4, CD84, FAM13A1, B4GT4, STAT3, G3BP2, EG1, FLJ13305, PODN, DNAH5, Dystrophin, GNPTA, MCT8, CARP, CTP synthase, C2CD4D, NHE3, MPV17L, IL-15, OLFML2A, C1orf88, CCDC39, DDHD1, H-

2L(d), Mca32, WIPI1, Trehalase, DPYD, Rab20, PDIP38, p200RhoGAP, SF3B14a, SPEF2, FLJ20898, OSF-2, REVERBalpha, HDAC11, 
NFKBIB, STOML3,

ZNF281, TR-alpha, MHC

Class I alpha chain, PNPase(old-35), GPR141, MEK1(MAP2K1), CTRL, C2, ARMC4, DOT1, SLIT3, C10orf58, PLB1, AM-R, DNAH1, PHEX, DNAJB11, SNX2, SP100, PVR, PLET1, ELMOD1, RIPK2, MDA-5, ASCT1 (SLC1A4), ZNF313, LYST, DENND1A, Hap-1, MGF, SNX1, MAD, PRKAR2B, ARG1, IL31RA, Olfactory receptor, Syntaxin 4, ARF6, LTBR1, TMEM2, SPAG17,

SCPEP1, CHMP6, Mdh1b, Guanylate cyclase, EPAS1, DMKN, Sec3, PRAM-1, Vinculin, APOD, EFP, Glycogen phosphorylase, HEY1, Vsm-RhoGEF, CEP78, Synaptotagmin II, ASCC3, VE-cadherin, NCKX3, Synaptotagmin, CD-MPR, CDPdiacylglycerol synthase, DHSO, HIST3H2A, Dynein, axonemal, light chains, PDE, IQCG, TREML2, MGAT4A, ACP33, KCNQ1OT1, PSMD8, Pleckstrin,

LRP12, Spermine oxidase, Guanylate cyclase alpha,

Galpha(q)-specific Class A Orphan/other GPCRs, Rab8B, HPLC2, CD300LG, Ephrin-A receptor 1, VIPAR, FLJ32810, ALPK1, NAT-1, HIF1A, Polycystin, LDB2, Fibulin5, Tmem195, RABL2A, FAT3, P2X7, I-kB, ORPfamily, NOTCH4, HES1, 
FLJ32658, Vitrin, MEK1/2, DGAT1, ATF-3, Olr1598, PPP4C, C20orf26, PAFAH gamma, MIRK, OLAH, MAPRE3(EB3), DENND1B, SAMHD1, VN1R4, B3GN1, DOK1, C3orf58, Egflam, ALKBH7, SLC24A6, ROBO1, DNAH9, Apaf-1, HAT1, JAK2, PLEKHM1, MR1, SGK3, ORP8, SMIF, GUCY1A2, DUS4L, ARHGEF19, ZCCHC14, PDE1, Islet-1, DMP4, Galpha(q)-specific nucleotidelike GPCRs, WDR93, PSMD3, FLVCR, DZIP1L, ATP6M, Rab-3, MIP-T3, Cytohesin1, MNS1, LRRC50, Ephrin-A receptor 5, MxA, FGFR3, FANCM, DNAH6, DYDC1, IDH1, RETSAT, SERPINB7, Ku70, Sortilin, MyHC, V-ATPase E subunit, OGFR, CD34, Spp24, Galectin-9, Ca-ATPase3, PCDHB8, Adipophilin, NHLRC2, PMCA2b, Carbohydrate sulfotransferases, Neuregulin 4, Ceb1, Scd2, YSK4, BBS9, PGS1, TRAP25, WHDC1, WRCH-1, Krm1, ATP1A2, Dynein, axonemal, heavy chains, RPGRIP1L, Oxygen-regulated protein 1, NNTM, CFA54, Alphaparvin, PKA-reg type II (cAMP-dependent), ENO4, CAP2, MYH10, ATP6V1B2, VANGL1, C4B protein, MANA, Profilin II, Lyn, PSMA1, Centaurin alpha 1, CENTA2, HLA-E, Ephrin-B2, 
DMPK, RNPEP, PRIC285,

MRE11, Ephrin-B, Dynein 2, cytoplasmic, heavy chain, PYGM, LMLN, NPX1, STRBP, CNDP2, PKI, CysLT2 receptor, RGS11, CDS2, TRIM15, G-protein alpha-14, CAPZA1, PKA-reg (cAMP-dependent), HA12 (rodent), ELL, CFA74, KIAA1340, VTA1, SPAG16, Alpha-actinin, CREG2, c-Maf, DNAH11, IL-15RA, GRO-3, TOP3A, RFX3, SLUG, MENA, PDE2A, RAP2C, ATP4A, Coagulation factor $\mathrm{X}$, Orexin receptor 1, A20, OASL, AZI2, ABBA, Ephrin-A receptors, GALRL, NPR3, GSTO1, Sp17, AKD1, KCNE4, KIF3B, Bim, SLC4A11, FAIM2, FNIP2, SHANK, Tryptase delta, SynGAP, Fibrinogen gamma, DAGK, PSMD14, IL-1 alpha, CREB3, CHST2, CCR1, CCR9, SULT1D1, RPS27, TFIIF, beta subunit, Hck, Hapln4, GMIP, TLR4, NAR4, C4, SCO2, NMT1, TMEM100, CYP2S1, Insulin, Fibulin-1, PBEF, PSMA2, GNT-IV, Ces1 (mouse), ADAR1, KIAA1377(HIP5), GRO-1, DUSP5, CD276, SPP lyase, TANK, Galectin-8, NT5C3, PHTF1, HYEP, PADI6, IDH2, SPATA18, CAPZA, CLR3, Asporin, FLJ46266, DSCR2, Notch, Orexin receptor, SLAM, APBA2BP, MCFD2, CAC, Galpha(q)specific peptide GPCRs, ISG20, PLEKHA5, Hun- 







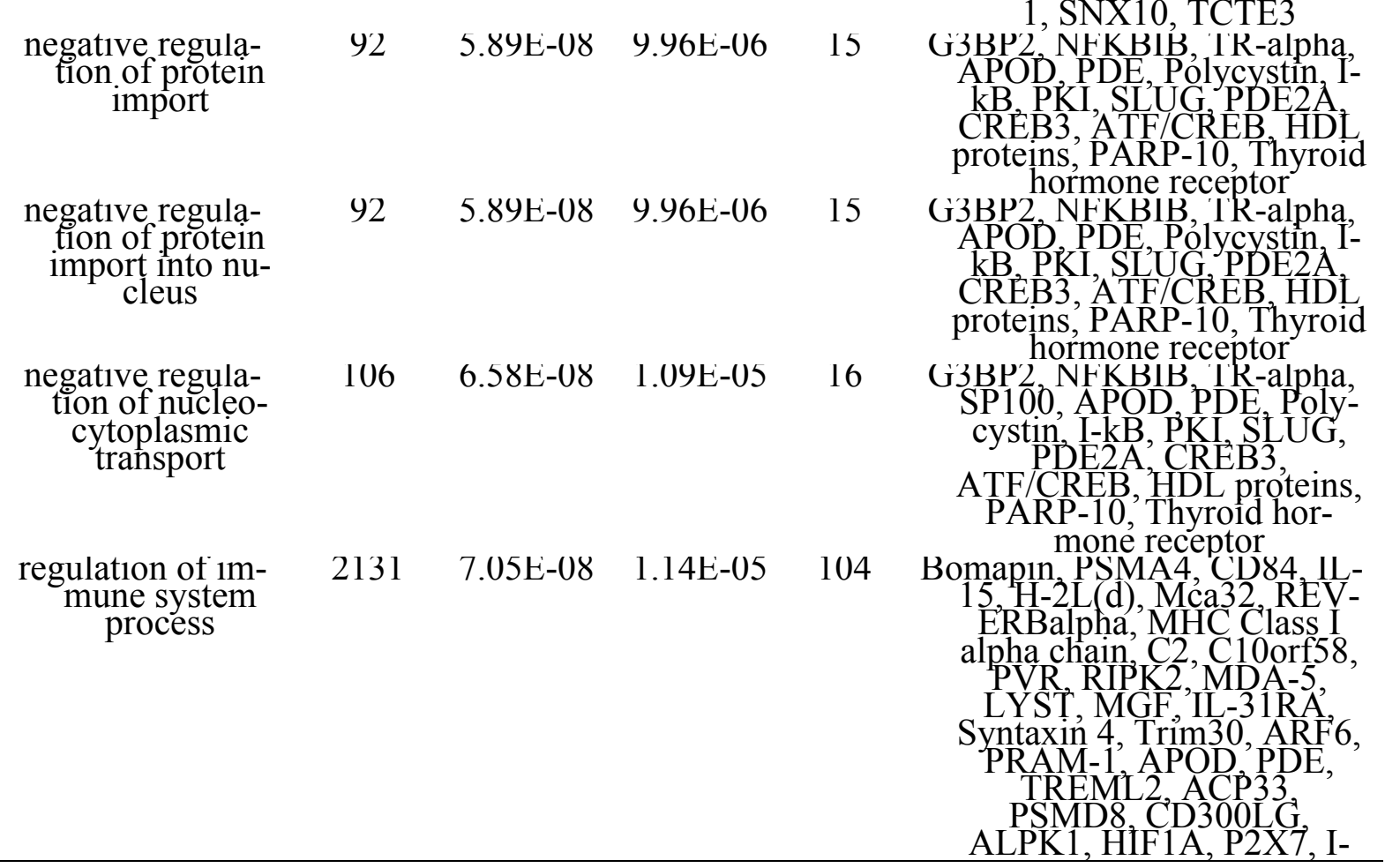


cellular developmental process
$4943 \quad 8.59 \mathrm{E}-08 \quad 1.34 \mathrm{E}-05 \quad 200$
KB, HEST, DENNDIB,

pha(q)-specific nucleotide-

like GPCRS, PSMD 3 MIP-

T3, FGFR3, MyHC, CD34,

Galectin-9, Carbohydrate

sulfotransferases, $\mathrm{Ceb} 1$

TRAFD1, CCDC 88, TFE3,

C4B protein. Lyn, PSMA1,

HLA-E, Ephrin-B2, EphrinB. TRIM15, PKA-reg (cAMP-dependent), HA12 (rodent), IL-15RA GRO-3, MENA, A20, PSMD14 IL1 alpha CREB3, CCR1, Hck, GMIP, TLR', C4 Insulin, PSMA2, ADAR1, GRO'-1, CD276, TANK' Galectin-8, BTNL2, Notch, SLAM, Galpha(q)-specific peptide GPCRs, CYFIP1, A11, CD 86, CCL27, CD40(TNFRSF5), PNPH, ATF/CREB, PSMD12, GITRL, HLA-C, HLA-B, CD209, HDL proteins, BCAP'MPP1 NCK1, USP18, IFNAR2, PLCL2, PI3K reg class IA CD300LF, TIG2. DDX 60,

IAT'3, FLJ13305, DNAH5, Dystrophin, GNPTA,

CARP IL-15, ${ }^{2} C D C 39, \mathrm{H}-$ 2L(d), PDIP38, SPEF2

FLJ20898, OSF-2, REV-

ERBalpha, HDAC11.

ZNF281,TR-alpha, MHC Class I alpha chain, PNPase old-35), MEK1(MAP2K1')

ARMC4, SLIT3, DNAHH1, SNX2, PLET1,ZNF313, Hap-1, MGF, SNX1, IL31RA, Olfactory receptor, ARF 6, SPAG17, EPAS1, DMKN, Vinculin, APOD,

HEY 1, Synaptotagmin II,' Synaptotagmin, Dynein, axonemal, light chains, PDE, IQCG, Pleckstrin, Rab8B, Ephrin-A receptor 1. VIPAR HIF1A

RABLL2A, FAT3, P2X7,

HES1, FLJ32658, MEK 1'2, ATF-3, MIRK, B3GN1, ROBO1, Apaf-1, JAK2, ORP8, PDE1, Islet-1,

DMP4, Galpha('))-specific nucleotide-like GPCRs.

FLVCR DZIP1L ATP6M,

Rab-3, MIP-T3, LRRC50, Ephrin-A receptor 5 , FGFR3, Ku70, Sortilin, MvHC CD34, Galectin-9, PMCA2b, Carbohydrate sulfotransferases, Ceb1, BBS9, Dynein, axonemal, heavy chains, RPGRIP1L, Oxygen-regulated protein 
Iocalization

6321

$8.71 \mathrm{E}-08 \quad 1.34 \mathrm{E}-05$

244
CAP2, MYHIO, Lyn, Ephrin-B2, Ephrin-B, Dynein 2 cytoplasmic, heavy chain, NPX1, STRBP, TRIM15, PKA'-reg (cAMP-dependent), HA12 (rodent), CFA74, SPAG16, Alpha-actinin, c-Maf, IL-

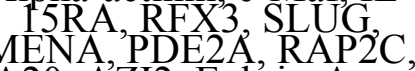
A20, A'ZI2, Ephrin-A receptors, FAtM', SHANK, SynGAP, Fibrinogen gamma, DA'GK, IL-1 alpha, CCR1, CCR9, Hck, TLR4, TMEM 100 , Insulin,

KIAA1377(HIP5), SPP 1yase, Galectin-8, IDH2 SPATA18, CAPZA, CLR3, FLJ46266, Notch, SLAM, Galpha(q)-specific peptide GPCRs, Huntingtin, GFRalphal, CYFIP1, HA11, CD86, Trp53inp2. HYDIN, Rab-3A, TSGA2, ACADM, CBR I, ES130 (p180), FGF10, T6441, ATF/CREB, ERK3,

DNAJC13, C40rf31, MAP1B, CCDC176, Glutaredoxin MMSA, HLA-C

DNÀI1, DZIPि, ZNF541, PDE1B, WDT2, RGS6, HLA-B, Artemis, CHD 7 , Alpha-actinin 3, HDL proteins, SIX5, Dynein, axonemal, intermediate

chains, NME5, SNAP-29,

Phosphatase regulator (inhibitor) OSTM1 (greyRANK(TNERSF11A),

KAD7, Protein p8, Thyroid hormone receptor, PLCL2, Nephrocystin-4, PTER, ABI2, TSNAXIP1,

CABYR, PI3K reg class

IA, CD300LF, TIG2, Perlecan, C2orf39, RSPH4A, NPNT, Alpha-internexin, FRK, SNX10

SLCU6A1, PAM, Clcal (rodent, CD84, STAT3, G3BP2, DNAH5, Dystrophin, GNPTA, MCT8, MPV 17L CCDC39.

Mca32, WIPI1, Rab20, CLCN7, NFKBIB, TRalpha, PNPase (old-35),

MEK1(MAP2K1), SLIT3, DNAH1, SNX2, PVR,

PLET1, ELMOD1, ASCT1 (SLC1A4), LYST

DENND1A, Hap-1, MGF,

SNX1, Olfạctory receptor, Syntaxin 4, ARF6, CHMP6, Guanylate cyclase, Sec3, Vinculin APOD, Synaptotagmin II, NCKX3, Synaptotagmin, 
CD-MPR, DHSO, Dynein, axonemal, light chains, PDE, Pleckstrin, LRP12 Galpha(q)-specific Class A Orphan/other GPCRs,

Rab8B, CD300LG, VIPÀR, Tbc1d9, HIF1A, SUSD2, Polycystin, Fibulin-5,

RABL2A, FAT3, P2X7, IkB, ORP-family, HES1, FLJ32658, MEK $1 / 2$, DGAT1. DENND1B, C3orf58, ALKBH7,

SLC24A 6, ROBO1, JAK2, PLEKHM1, SGK 3, ORP 8, SMIF, Islet-1, Galpha(q)specific nucleotide-like GPCRs, FLVCR, ATP6M, Rab-3, GOLT1A, MIP-T3, Cytohesin1, FAM38B,

DNAH6, SERPINB7, Sor-

tilin, MyHC, V-ATPase E subunit, CD34, Spp24, Galectin-9, Ca-ATPase3, Adipophilin, PMCA2b, Carbohydrate sulfotransferases, BBS9 C9 orf72. UGRP1, WHDC1, ATP1A2,

Dynein, axonemal, heavy chains, NNTM, CFA54, Alpha-parvin, ENO4.

MYH10, ATP6V1B2, C4B protein, Lyn, Ephrin-B2, CCDC46, Ephrin-B, Dynein 2, cytoplasmic heavy chain, hRap1, NPX1,

NIPA2, VTA1, SPAG16, Bestrophin-4, Alpha-

actinin, DNAH11, GRO-3, MEX3D, RFX3, SLUG,

Coagulation factor $\mathrm{X}, \mathrm{A} 20$, Ephrin-A receptors, NPR3, Sp17, KCNE4, KIF3B, Bim, SLC4A11, SHANK, SynGAP, Fibrinogen gam-

ma, DAGK, CREB3, PLEKHM2 2 C4, $\mathrm{SCO} 2$, NMT1, Insulin, ADARI, GRO-1, SPP lyase, PADI6, IMP2 CLR3, Asporin.

Notch, SLAM, APBA2BP,

MCFD2, CAC, Galpha(q)specific peptide GPCRs, Huntingtin GFRalphal, CYFIP 1, Mitd1, Profilin, DHX38, ES130'(p180), FGF10, PNPH ATF/CREB, DNAJC13, EPS8 C40rf31 SCN3A, DNAH3, MÀP-1B,

SERINC5, CD163 FADS2, Glutaredoxin, DNAH2, PEAR1, ATP6V1B, CCDC52, DNAI1, DZIP, myristoyltransferase, ATP6V1E, MARCH5, 


\section{axonemal dynein complex as- sembly} regulation of in-
nate immune
response

regulation of defense response

\section{$1.11 \mathrm{E}-07 \quad 1.68 \mathrm{E}-05$}

514

$2.05 \mathrm{E}-05$

38

\section{$1.48 \mathrm{E}-07 \quad 2.05 \mathrm{E}-05$}

mediate chains, FAT1, 29. Phosphatase regulator (inhibitor), OSTMP (greylethal), Syntenin 1 ATP8B3, VAMP4, ILPIP, NCK 1

RANK(TNFRSF11A),

P2Y4, Beta adducin, Thyroid hormone receptor, BET1, NXT2, Nephrocystin-4, GPP130, ABI2, RPESP, LULL1, PI3K reg class IA, Galpha(s)-specific peptide GPCRs, DUSP18, TIG2, Perlecan, C2orf39, RNF22, PSTPIP2, Glutaredoxin 1, SNX10,

DNAH5 CCDC39, ARMC4, DNAH1, LRRC50, Dynein, axonemal, heavy chains,

DNAI1, Dynein, axonemal, intermediate chains, C2orf39

PSMA4, REV-ERBalpha, MHC Class I alpha chain, PVR, RIPK2, MDA-5,

Trim30, ARF6, PSMD8, IkB SAMHD1, JAK2, PSMD3, Galectin-9,

TRAFD1,Lyn PSMA1, HLA-E, TRIM15, A20, PSMD 14, CCR1, Hck, TLR4, Insulin, PSMA2, ADAR1, TANK, Gạlpha(q)-specific peptide GPCRS, CD86, PSMD12, USP18, IFNAR2, DDX60, SERPINB9

Bomapin, PSMA4 IL-15, WFDCl', REV-ERBalpha, MHC Class I alpha chain, C2, PVR, RIPK 2, MDA-5, Trim30, ARF6, APOD, Synaptotagmin, PDE,

PSMD8, Galpha(q)-specific Class A Orphan/other

GPCRs, ALPK1, I-kB, Galpha(q)-specific nucleotide-like GPCRs, PSMD3, MIP-T3, Galectin-9, Carbohydrate sulfotransferases, Ceb1, TRAFD1, C4B protein, Lyn, PSMA1, HLA-E, TRIM1'5, PDE2À A20, SHANK, PSMD14, CREB3 CCR 1, Hck GMIP, TLR 4, C4, Insulin, Pyrin'(MEFV), PSMA2, ADAR1, CD276, TANK, Galpha(g)-specific peptide GPCRs, CD86

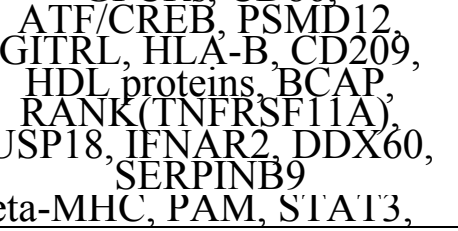




\begin{tabular}{|c|c|}
\hline process & 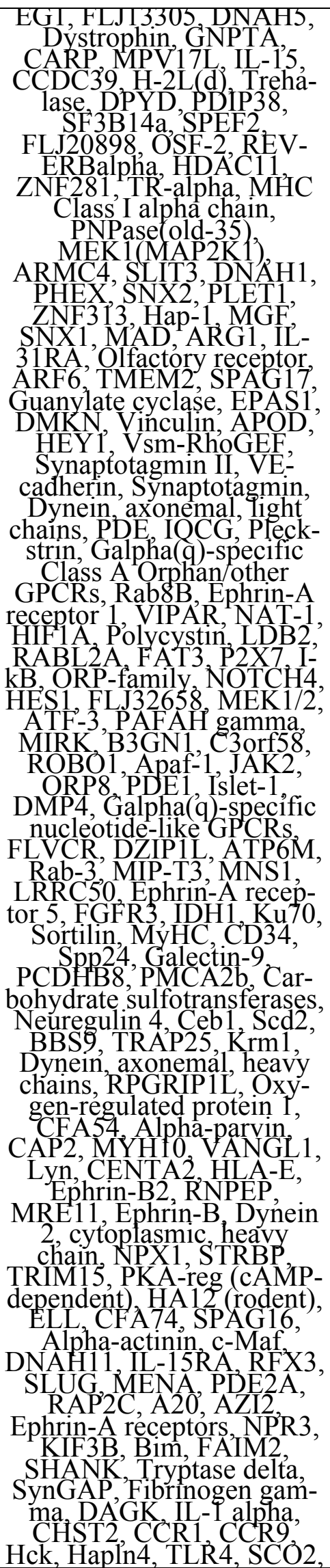 \\
\hline
\end{tabular}


crlium-dependent cell motility
16

$1.49 \mathrm{E}-07 \quad 2.05 \mathrm{E}-05$

NMT1, TMEMTO0, Insulin,

Fibulin-1, PBEF, ÅDAR1, KIAA 1377(HIP5), GRO-1,
DUSP5, SPP lyase, Galectin-8, HYEP, IDH2, IMP2,

SPATA18, CAPZA, CLR3, Asporin, FLJ46266. DSCR2, Notch, SLAM, MCFD2, Galpha(q)specific peptide GPCRs, PLEKHA5, Huntingtin, GFRalpha1, CYFIP1

HA11 CD86, POLR2G, Trp53inp2, Profillin,

HYDIN, Rab-3A, TSGA2, ES130 (p180), FGF10,

CD40(TNFRSF5), T6441, ATF/CREB, ERK3, DNAJC13, C40rf31, SCN3A, MAP-1B SERINC5, CCDC176, SETDB2, Ġlutaredoxin,

MMSA, HLA-C,
ATP6V1B, DNAI1,
SHANK2, AGAL, DZIP, ZNF541 ÅL3A1, PDE1B,
WDT2, N-

myristoyltransferase,
PCDHB16, PIP1, RGS6,

HLA-B, Artemis, CHD7, Alpha-actinin 3, HDL proteins, SIX5, Dynein, axonemal, intermediate chains, C9orf98, FAT1, phatase regulator (inhibitor) OSTM1 (grey-lethal, TRIML1, UGCGG IRGUK,

RANK(TNFRSF11Ả), Beta adducin, GREM2, KÀD7, Protein p8, AL1A1, Thyroid hormone receptor, PLCL 2, Nephrocystin-4, Lrig3,PTER, STOX2, ABI2, TSNAXIP1, CABYR, PI3K reg class IA, RGS9, CD300LF, Galpha(s)-specific peptide GPCRs, TIG2, Perlecan, Alpha-internexin, FRK, RNF22, Glutaredoxin 1, SNX10

CCDC39, DNAH1, Dynein, axonemal, heavy chains, CFA54, SPAG16, RFX3, C2orf39

\section{References for Supplementary Material}

Chandler, J.D., et al. (2016) Metabolic pathways of lung inflammation revealed by highresolution metabolomics (HRM) of H1N1 influenza virus infection in mice. Am J Physiol Regul Integr Comp Physiol, 311, R906-R916. 
XMWAS: $R$ package for data integration and network analysis

Fortin, G., et al. (2009) L-carnitine, a diet component and organic cation transporter OCTN ligand, displays immunosuppressive properties and abrogates intestinal inflammation. Clin Exp Immunol, 156, 161-171.

Kohlmeier, J.E. and Woodland, D.L. (2009) Immunity to respiratory viruses. Annu Rev Immunol, 27, 61-82.

Liu, Q. et al. (2016) The cytokine storm of severe influenza and development of immunomodulatory therapy. Cell Mol Immunol, 13, 3-10.

Miyake, J.H. et al. (2000) Bile acid induction of cytokine expression by macrophages correlates with repression of hepatic cholesterol 7alpha-hydroxylase. J Biol Chem, 275, 21805-21808.

Sadik, C.D. and Luster, A.D. (2012) Lipid-cytokine-chemokine cascades orchestrate leukocyte recruitment in inflammation. J Leukoc Biol, 91, 207-215.

Shannon P. et al. (2003) Cytoscape: a software environment for integrated models of biomolecular interaction networks. Genome Res, 13, 2498-2504.

Yin, K. and Agrawal, D.K. Vitamin D and inflammatory diseases. (2014) J Inflamm Res 7, 69-87. 